\title{
Mordell-Weil torsion and the global structure of gauge groups in F-theory
}

\author{
Christoph Mayrhofer, ${ }^{a}$ David R. Morrison, ${ }^{b}$ Oskar Till ${ }^{a}$ and Timo Weigand ${ }^{a}$ \\ ${ }^{a}$ Institut für Theoretische Physik, Ruprecht-Karls-Universität, \\ Philosophenweg 19, 69120, Heidelberg, Germany \\ ${ }^{b}$ Departments of Mathematics and Physics, \\ University of California Santa Barbara, Santa Barbara, U.S.A. \\ E-mail: c.mayrhofer@thphys.uni-heidelberg.de, drm@math.ucsb.edu, \\ o.till@thphys. uni-heidelberg.de, t. weigand@thphys.uni-heidelberg.de
}

ABSTRACT: We study the global structure of the gauge group $G$ of F-theory compactified on an elliptic fibration $Y$. The global properties of $G$ are encoded in the torsion subgroup of the Mordell-Weil group of rational sections of $Y$. Generalising the Shioda map to torsional sections we construct a specific integer divisor class on $Y$ as a fractional linear combination of the resolution divisors associated with the Cartan subalgebra of $G$. This divisor class can be interpreted as an element of the refined coweight lattice of the gauge group. As a result, the spectrum of admissible matter representations is strongly constrained and the gauge group is non-simply connected. We exemplify our results by a detailed analysis of the general elliptic fibration with Mordell-Weil group $\mathbb{Z}_{2}$ and $\mathbb{Z}_{3}$ as well as a further specialization to $\mathbb{Z} \oplus \mathbb{Z}_{2}$. Our analysis exploits the representation of these fibrations as hypersurfaces in toric geometry.

KEYwORDS: F-Theory, D-branes

ARXIV EPRINT: 1405.3656 


\section{Contents}

1 Introduction $\quad 2$

2 The arithmetic of elliptic fibrations 3

2.1 The Mordell-Weil group 4

2.2 Elliptic fibrations with torsion Mordell-Weil group 4

3 F-theory fibrations with non-trivial Mordell-Weil group $\quad 6$

3.1 The free Mordell-Weil group and the Shioda map $\quad 7$

$\begin{array}{lll}3.2 & \text { Torsional sections and divisor classes } & 7\end{array}$

3.3 The global structure of the gauge group in presence of Mordell-Weil torsion $\quad 9$

4 Mordell-Weil group $\mathbb{Z}_{2} \quad 12$

$\begin{array}{lll}4.1 & \mathrm{An} \mathrm{SU}(2) / \mathbb{Z}_{2} \text {-fibration } & 12\end{array}$

$\begin{array}{lll}\text { 4.1.1 Singularity structure and resolution } & 12\end{array}$

$\begin{array}{ll}\text { 4.1.2 Torsional divisors and free quotient } & 16\end{array}$

$\begin{array}{llr}4.2 & \text { An }(\mathrm{SU}(2) \times \mathrm{SU}(2)) / \mathbb{Z}_{2} \text {-fibration } & 18\end{array}$

4.3 An $(\mathrm{SU}(4) \times \mathrm{SU}(2)) / \mathbb{Z}_{2}$-fibration $\quad 21$

4.4 A $(\operatorname{Spin}(7) \times \mathrm{SU}(2)) / \mathbb{Z}_{2}$-fibration 22

4.5 Generalisation to $\operatorname{Sp}(n) / \mathbb{Z}_{2}, \mathrm{SU}(2 n) / \mathbb{Z}_{2}, \operatorname{Spin}(4 n) / \mathbb{Z}_{2}$, Type IIB limit and restricted monodromies 24

$5 \quad$ Mordell-Weil group $\mathbb{Z} \oplus \mathbb{Z}_{2} \quad 26$

5.1 An $(\mathrm{SU}(2) \times \mathrm{SU}(2)) / \mathbb{Z}_{2} \times \mathrm{U}(1)$ fibration 26

5.2 A chain of fibrations via Higgsing $\quad 29$

5.3 An $(\mathrm{SU}(4) \times \mathrm{SU}(2) \times \mathrm{SU}(2)) / \mathbb{Z}_{2} \times \mathrm{U}(1)$ fibration 30

6 Mordell-Weil group $\mathbb{Z}_{3} \quad 33$

6.1 An SU(3)/Z्Z 3 -fibration 33

6.2 An $(\mathrm{SU}(6) \times \mathrm{SU}(3)) / \mathbb{Z}_{3}$-fibration 36

$\begin{array}{lll}7 & \text { Conclusions } & 39\end{array}$

$\begin{array}{ll}\text { A More on fiber structures } & 40\end{array}$

$\begin{array}{lll}\text { A.1 } & \mathfrak{s u}(4) \text { top over polygon } 13 & 40\end{array}$

A.1.1 Codimension one 40

A.1.2 Codimension two 40

$\begin{array}{lll}\text { A.2 } & \mathfrak{s u}(4) \text { top over polygon } 15 & 41\end{array}$

A.2.1 Codimension one 41

A.2.2 Codimension two 41

$\begin{array}{lll}\text { A.3 } & \mathfrak{s u}(6) \text { top over polygon } 16 & 42\end{array}$

A.3.1 Codimension one $\quad 42$

A.3.2 Codimension two 42 


\section{Introduction}

The Mordell-Weil group of rational sections of an elliptic fibration has attracted a great deal of interest in the recent F-theory literature. The free part of the Mordell-Weil group encodes information about the abelian sector of an F-theory model [1-3]. Explicit realizations of $\mathrm{U}(1)$ gauge groups via such rational sections in F-theory have been studied in detail [4-22]. ${ }^{1}$ In this paper we investigate the role of the torsion part of the Mordell-Weil group and its relation to global properties of the non-abelian gauge sector of the F-theory vacuum.

Non-abelian gauge symmetries in F-theory have their origin in the codimension-one singularity structure. The by now algorithmic procedure to engineer gauge theories takes the Kodaira classification of singular fibers as the starting point. However, the resolution of codimension-one singularities provides only information on the gauge algebra, and not on the gauge group. The Lie group whose Lie algebra is given by the geometric data might be simply connected, making the lift of the Lie algebra and its representations trivial. If it is non-simply connected only a subset of the matter representations will be present in the gauge theory. The difference between such theories is in particular measured by non-local operators, see e.g. [29]. For the example of the Standard Model, the gauge group is presumably not the simply connected $\mathrm{SU}(3)_{c} \times \mathrm{SU}(2)_{W} \times \mathrm{U}(1)_{Y}$ but really $\left(\mathrm{SU}(3)_{c} \times \mathrm{SU}(2)_{W} \times \mathrm{U}(1)_{Y}\right) / \mathbb{Z}_{6}$, where $\mathbb{Z}_{6}$ is a subgroup of the center $\mathbb{Z}_{3} \oplus \mathbb{Z}_{2} \oplus \mathrm{U}(1)$, and only matter multiplets invariant under the action of $\mathbb{Z}_{6}$ are present [30]. Indeed, embedding the Standard Model into SU(5) amounts to choosing a block diagonal decomposition $S(\mathrm{U}(2) \times$ $\mathrm{U}(3)) \subset \mathrm{SU}(5)$ such that its determinant is unity, and $S(\mathrm{U}(2) \times \mathrm{U}(3))$ is isomorphic to $\left(\mathrm{SU}(3)_{c} \times \mathrm{SU}(2)_{W} \times \mathrm{U}(1)_{Y}\right) / \mathbb{Z}_{6}$, see e.g. [31].

The global properties of a gauge group are related to torsion elements of the MordellWeil group. The study of torsional sections in F-theory fibrations (i.e. sections of the fibration which induce torsion elements in the Mordell-Weil group) was initiated in [32]. By utilizing the duality between F-theory and heterotic theory in eight dimensions it was shown that the fundamental group of the gauge group is isomorphic to the torsion subgroup of the Mordell-Weil group, and it was conjectured that the same result holds for six-dimensional compactifications. The general framework relating the Mordell-Weil group of the fibration to the gauge group of F-theory was laid out in [33]. The Mordell-Weil group has also been studied via string junctions and configurations of $(p, q)$-branes [34]. This approach was eight-dimensional and reproduces the classification of Mordell-Weil lattices for elliptic surfaces [35, 36]. Subsequent work addressed the same problem for elliptic threefolds [37].

In this article, we show how the global structure of the gauge theory manifests itself for F-theory in any dimension. Rather than relying on heterotic duality or the physics of string junctions, we directly study the effect of a torsional Mordell-Weil subgroup on the physics of the F-theory compactification with geometric means. Our starting point is a generalization of the Shioda map [38-40] to torsional sections. Unlike for non-torsional sections, this map defines a trivial divisor class on the elliptic variety. We use this class to construct an element in the coweight lattice which takes integer values on any charged matter representation

\footnotetext{
${ }^{1}$ This has to a large extent been motivated from model building and the need for abelian selection rules in GUT models, see e.g. [23-28] for a rather incomplete list of references.
} 
that can occur in the compactification. The coweight in question is associated with a fractional linear combination of the resolution divisors which correspond to the Cartan generators of the gauge algebra. The requirement that this fractional linear combination must have integer pairing with the matter representations strongly constrains the set of admissible representations. As a result, the center of the gauge group is smaller compared to naive expectations and the gauge group acquires a non-trivial first fundamental group. The divisor associated with the coweight is a torsional element of $H^{1,1}(\hat{Y}, \mathbb{Z})$ modulo the resolution divisors associated with the gauge algebra realized on the elliptic fibration $\hat{Y}$. This clarifies the relation between torsion in the Mordell-Weil group and torsion in the cohomology group of the elliptic fibration.

To exemplify this general structure we explicitly analyze F-theory compactifications on elliptic fibrations whose fiber can be realized as a hypersurface in a toric ambient space. Out of the 16 possible toric realizations of such elliptic fibrations, three are known to have torsional Mordell-Weil group $\mathbb{Z}_{2}, \mathbb{Z}_{3}$ and $\mathbb{Z} \oplus \mathbb{Z}_{2}[15]$. We will show that the first two correspond, in fact, to the most general elliptic fibrations with Mordell-Weil group $\mathbb{Z}_{2}$ and $\mathbb{Z}_{3}$ in the list presented in [32], while the $\mathbb{Z} \oplus \mathbb{Z}_{2}$ model is a restriction of the $\mathbb{Z}_{2}$ fibration. Certain blow-downs of these fibrations have also been considered previously in [41] as examples of elliptic fibrations with restricted $\operatorname{SL}(2, \mathbb{Z})$-monodromy. The fibrations we consider allow for a representation as a global Tate model and can be obtained as a special case of the $\mathrm{U}(1)$ restricted Tate model [4]. The restriction of the complex structure of the fibration necessary to implement torsion in the Mordell-Weil group automatically induces non-abelian singularities in codimension one, which we resolve and study in detail. The associated gauge group factor can be viewed as the non-abelian enhancement of the $\mathrm{U}(1)$ gauge group in the underlying $\mathrm{U}(1)$ restricted Tate model, to which the geometries are consequently related by a chain of (un)Higgsings. Furthermore, we exemplify the construction of extra non-abelian gauge group factors via toric tops [42, 43]. The possible extra gauge group factors follow a specific pattern dictated by the torsional sections. As predicted by our general analysis of Mordell-Weil torsion, only a subset of typically realized matter representations is present in the geometry.

In section 2 we begin with a brief review of the Mordell-Weil group with special emphasis on its torsion subgroup. In section 3 we outline the general picture of our geometric construction of the coweight lattice and elucidate the relation between the Mordell-Weil group and global properties of the gauge group in F-theory. Our exemplification of these general results for elliptic fibrations with Mordell-Weil torsion $\mathbb{Z}_{2}, \mathbb{Z} \oplus \mathbb{Z}_{2}$ and $\mathbb{Z}_{3}$ follows in sections 4,5 and 6 , respectively. Some computational details are relegated to the appendix.

\section{The arithmetic of elliptic fibrations}

In this section, we give a brief review of the Mordell-Weil group of a family of elliptic curves. We describe how meromorphic sections naturally come with a group structure and comment in particular on the finite part of this group, the part associated to "torsional sections." This is a classic topic in mathematics and for more extensive treatments see e.g. $[44,45]$. 


\subsection{The Mordell-Weil group}

An elliptic curve $E$ is a smooth complex curve of genus one with a marked point. Such a curve may be given in Weierstrass form

$$
y^{2}=x^{3}+f x z^{4}+g z^{6}
$$

with coordinates $[x: y: z] \in \mathbb{P}_{2,3,1}^{2}$ and $f, g$ valued in some field $K$. For fixed values of $f$ and $g$ this genus one curve is the flat torus given by the quotient

$$
E=\frac{\mathbb{C}}{\Lambda}
$$

of the complex plane $\mathbb{C}$ by $\Lambda=\langle 1, \tau\rangle$, i.e. the lattice generated by 1 and $\tau$. These two descriptions are equivalent and for $z \in \mathbb{C}$ the isomorphism is given $b^{2}$

$$
z \mapsto\left[\wp(z): \wp^{\prime}(z): 1\right]
$$

where $\wp$ is the doubly periodic Weierstrass function. The complex structure parameter $\tau$ is related to the Weierstrass equation via the modular $j$-function

$$
j(\tau) \sim \frac{f^{3}}{4 f^{3}+27 g^{2}} .
$$

Because of the isomorphism (2.3) the addition of complex numbers in $\mathbb{C} / \Lambda$ induces an addition of points on the curve (2.1). The set of rational points on $E$, i.e. points given by rational expressions in the field $K$, is closed under this addition and thus forms an abelian group. This group is often denoted by $E(K)$ and the abelian structure makes elliptic curves examples of abelian varieties. The original Mordell-Weil theorem states that this group is finitely generated when $K$ is a "number field", i.e. a finite extension of the rational numbers. In this case,

$$
E(K)=\mathbb{Z}^{r} \oplus \mathbb{Z}_{k_{1}} \oplus \cdots \oplus \mathbb{Z}_{k_{n}} .
$$

The rank $r$ of this group is the number of generators of the free subgroup and the finite part is called the torsion subgroup $E(K)_{\text {tors. }}$ A theorem by Mazur states that for a curve over the rationals, the torsion subgroup $E(\mathbb{Q})_{\text {tors }}$ is either $\mathbb{Z}_{k}$ for $k=1, \ldots, 10,12$ or $\mathbb{Z}_{2} \oplus \mathbb{Z}_{k}$ for $k=2,4,6,8$. The converse statement also holds, i.e. all possibilities are realised.

\subsection{Elliptic fibrations with torsion Mordell-Weil group}

The notion of the Mordell-Weil group also applies to families of elliptic curves, i.e. fibrations

$$
\pi: Y \rightarrow \mathcal{B}
$$

with a distinguished zero-section $\sigma_{0}$ such that the fiber $\pi^{-1}(b)$ for a generic point $b \in \mathcal{B}$ is an elliptic curve. We can regard the coefficients of the Weierstrass equation (2.1) as taking values in the field $K$ of meromorphic functions on the base $B$. Each meromorphic section of

\footnotetext{
${ }^{2}$ Actually, this parametrisation corresponds to the Weierstrass equation $y^{2}=4 x^{3}+f x+g$, but this difference will not be of interest for our purposes.
} 
the fibration determines an element of $E(K)$, because it detemines $x=x(b)$ and $y=y(b)$, the "coordinates" of the point, as elements of the field $K$ of meromorphic functions. The zero-section $\sigma_{0}$ maps to the identity element in the group $E(K)$, and the group structure is given by fiberwise addition of points.

The "Mordell-Weil theorem for function fields" (proved by Lang and Néron [46]) says that in this situation, $E(K)$ is also finitely generated unless the fibration is "split", i.e. unless $Y$ is birational to a product $E \times \mathcal{B}$. Note that the zero-section does not serve as one of the generators of the group. In particular, the Mordell-Weil group is trivial when the zero section is the only section of the fibration, and extra rational sections are needed to have a non-trivial group. For certain elliptic surfaces the possible groups $E(K)$ have been classified analogously to the Mazur theorem for elliptic curves. For instance, for a rational elliptic surface the non-trivial possibilities for the Mordell-Weil group are

$$
\begin{array}{lll}
\mathbb{Z}^{r}(1 \leq r \leq 8), & \mathbb{Z}^{r} \oplus \mathbb{Z}_{2}(1 \leq r \leq 4), & \mathbb{Z}^{r} \oplus \mathbb{Z}_{3}(1 \leq r \leq 2), \\
\mathbb{Z}^{r} \oplus \mathbb{Z}_{2} \oplus \mathbb{Z}_{2}(1 \leq r \leq 2), & \mathbb{Z} \oplus \mathbb{Z}_{4}, & \mathbb{Z}_{2} \oplus \mathbb{Z}_{4}, \\
\mathbb{Z}_{2} \oplus \mathbb{Z}_{2}, & \mathbb{Z}_{3} \oplus \mathbb{Z}_{3}, & \mathbb{Z}_{k}(2 \leq k \leq 6)
\end{array}
$$

and in particular the Mordell-Weil group for any rational elliptic surface is torsion-free if its rank is greater than 4 [35]. For elliptic K3 surfaces the list is more complicated, but completely known [36]; in particular, the possibilites for non-trivial torsion in the MordellWeil group are

$$
\mathbb{Z}_{k}(2 \leq k \leq 8), \quad \mathbb{Z}_{2} \oplus \mathbb{Z}_{2 k}(1 \leq k \leq 3), \quad \mathbb{Z}_{3} \oplus \mathbb{Z}_{3}, \quad \mathbb{Z}_{4} \oplus \mathbb{Z}_{4} .
$$

The general situation for higher-dimensional fibrations, e.g. three- and fourfolds, is not as well understood and classifications only exist in special cases such as [47].

A useful tool to study in particular higher-dimensional examples of elliptic fibrations is toric geometry. In toric geometry an elliptic curve may be realized as a hypersurface or a complete intersection in a toric ambient space. The possible realizations as hypersurfaces are classified by the 16 reflexive polygons in two dimensions. The associated toric ambient spaces are $\mathbb{P}_{1,1,2}^{2}, \mathbb{P}^{1} \times \mathbb{P}^{1}, \mathbb{P}^{2}$ or blow-ups thereof. Three of these polygons admit torsional sections given as the intersection of an ambient toric divisor with the elliptic curve. According to the enumeration of polygons in [43], the elliptic curves in the ambient spaces defined by polygon 13,15 and 16 have toric Mordell-Weil groups $\mathbb{Z}_{2}, \mathbb{Z} \oplus \mathbb{Z}_{2}$ and $\mathbb{Z}_{3}$, respectively [15] (see also [48]). These cases will be studied in detail in this paper including the toric implementation of further non-abelian gauge groups via tops.

An important ingredient in our analysis is the correspondence between rational sections and certain divisor classes on the fibration, more precisely elements of the Néron-Severi group of divisors modulo algebraic equivalence. Note that the Néron-Severi group coincides with the Picard group of divisors modulo linear equivalence for spaces with vanishing first cohomology group, which is the situation of relevance throughout this paper. ${ }^{3}$ Let $E$ be a general fiber of $\pi$. Each divisor $D$ on $Y$ can be restricted to a divisor $\left.D\right|_{E}$ on $E$ which has

\footnotetext{
${ }^{3}$ For this reason, we will systematically restrict our notation to refer to the Néron-Severi group rather than the Picard group.
} 
a specific degree $D \cdot E$. For example, sections restrict to divisors of degree 1 . Now for an arbitrary divisor $D$, the linear combination $D-(D \cdot E) \sigma_{0}$ restricts to a divisor of degree 0 on $E$. But the set of divisors of degree 0 on $E$ is just $E$ itself.

In this way, we get a surjective homomorphism of groups

$$
\psi: N S(Y) \rightarrow E(K)
$$

which sends $[D]$ to the $K$-valued point of $E$ determined by restricting the divisor $D-(D$. $E) \sigma_{0}$ to $E$. (It is surjective because every element of $E(K)$ arises from a rational section $\sigma$.) The kernel of this homomorphism is generated by the zero section and by divisors whose restriction to the general fiber $E$ is trivial.

Recall that the elliptic fiber degenerates when the discriminant $\Delta=4 f^{3}+27 g^{2}$ vanishes. The singularities, if any, in the total space of $Y$ can always be resolved, and $\mathcal{B}$ can be further blown up if, necessary, to ensure a birational model $\hat{\pi}: \hat{Y} \rightarrow \hat{\mathcal{B}}$ of our fibration with a nonsingular total space $\hat{Y}$ and a flat fibration, i.e. a fibration in which all of the fibers are one-dimensional. In the sequel we assume that our original base $\mathcal{B}$ allows for a resolution $\hat{Y}$ which is nonsingular and has a flat family. The resolution process introduces a set $F_{i}$ of resolution divisors which are $\mathbb{P}^{1}$-fibrations over the codimension-one locus in the base $\mathcal{B}$ over which the singularity was located. Let $\mathcal{T}$ denote the subgroup of $N S(\hat{Y})$ generated by the zero-section $\left[\sigma_{0}\right]$, the resolution divisors $F_{i}$, and divisors of the form $\pi^{-1}(\delta)$ for $\delta \in N S(\mathcal{B})$. The Shioda-Tate-Wazir theorem [39, 49] asserts that the kernel of the map $\psi$ in $(2.9)$ is $\mathcal{T}$. In particular,

$$
\operatorname{rank} N S(\hat{Y})=1+\operatorname{rank} N S(\mathcal{B})+\operatorname{rank} E(K)+\sum_{w \in \Delta}\left(n_{w}-1\right),
$$

where $n_{w}$ is the number of irreducible components of the resolved fiber over the codimension-one loci $w \in \Delta \subset \mathcal{B}$ over which the fiber degenerates.

The divisors on $\hat{Y}$ are thus generated by the class of the zero section $Z=\left[\sigma_{0}\right]$, the pullback of divisors in $\mathcal{B}$, the divisor classes $S_{i}-Z=\left[\sigma_{i}\right]-\left[\sigma_{0}\right]$ from the free generators of $E(K)$ and the irreducible fiber resolution divisors $F_{i}$. On the other hand the divisor class $R-Z=\left[\sigma_{r}\right]-\left[\sigma_{0}\right] \in N S(\hat{Y})$ associated with a torsional section $\sigma_{r}$ has the property that $k(R-Z)$ can be expressed in terms of the generators of $\mathcal{T}$, where $k$ is the order of the torsional element of the Mordell-Weil group. It follows that $R-Z$ can be expressed in terms of these generators using $\mathbb{Q}$-coefficients. As described in the next section, this expression for $R-Z$ is closely related to the so-called Shioda map [38-40]. This is in line with the result for elliptic surfaces in [50], where a trivial class on the hypersurface is obtained by adding a certain rational linear combination of resolution divisors to $R-Z$.

\section{F-theory fibrations with non-trivial Mordell-Weil group}

After a brief review of the physics of the free Mordell-Weil group and abelian gauge symmetries, a subject treated in great detail in the recent F-theory literature, we outline the general picture of torsional sections and the global structure of the gauge theory. 
In the sequel we denote by $G$ the non-abelian part of the gauge group of an F-theory compactification on an elliptically fibered Calabi-Yau 4 -fold $Y_{4}$ over the base manifold $\mathcal{B}$ and denote its Cartan subgroup by $H$. Let us assume that the singularities of $Y_{4}$ responsible for the appearance of a non-abelian gauge group $G$ in codimension-one admit a crepant resolution $\hat{Y}_{4}$. Expanding the M-theory 3 -form $C_{3}$ as $C_{3}=\sum_{i} A_{i} \wedge F_{i}$ with $F_{i}$ the resolution divisors gives rise to the Cartan $\mathrm{U}(1)$ gauge fields $A_{i}$. Therefore the resolution divisors $F_{i}$ span the coroot lattice $Q^{\vee}$ of the Cartan subalgebra $\mathfrak{h}$.

\subsection{The free Mordell-Weil group and the Shioda map}

Since the group homomorphism (2.9) is surjective, there is an injective homomorphism in the other direction after tensoring with $\mathbb{Q}$. In the case of elliptic surfaces, Shioda [38] introduced such a homomorphism with a specific additional property, which was extended in $[39,40]$ to a Shioda map for elliptic fibrations of arbitrary dimension. For an elliptic fourfold $\hat{Y}_{4}$, the Shioda map

$$
\varphi: E(K) \rightarrow N S\left(\hat{Y}_{4}\right) \otimes \mathbb{Q}
$$

satisfies the property that $\langle\varphi(\sigma), T\rangle=0$ for any divisor $T \in \mathcal{T}$, where the pairing $\langle$,$\rangle is$ the height pairing

$$
\left\langle D_{1}, D_{2}\right\rangle:=\pi\left(D_{1} \cap D_{2}\right),
$$

which projects the intersection of two divisors to the base. It is well defined modulo linear equivalence, and so defines a pairing on the Néron-Severi group. For example, given any section $S$ defining an element $S-Z$ of the Mordell-Weil group, we have

$$
\varphi(S-Z)=S-Z-\pi^{-1}(\delta)+\sum l_{i} F_{i}
$$

for some divisor $\delta$ on $\mathcal{B}$ and some rational numbers $l_{i} \in \mathbb{Q}$, which is constructed so that for every $T \in \mathcal{T}$ we have

$$
\pi\left(T \cap\left(S-Z-\pi^{-1}(\delta)+\sum l_{i} F_{i}\right)\right)
$$

is linearly equivalent to zero on the base $\mathcal{B}$.

Let us denote by $\mathcal{S}$ the harmonic 2 -form representative of the cohomology class associated with $\varphi(S-Z)$. Expanding the M-theory 3 -form as $C_{3}=A_{\mathcal{S}} \wedge \mathcal{S}$ gives $A_{\mathcal{S}}$ as a massless $\mathrm{U}(1)$ one-form gauge field in three dimensions. ${ }^{4}$ The details of the map assert that this generator does not lie in the Cartan of any non-abelian gauge symmetry, and that it has 'one leg in the fiber', ensuring that the gauge field $A_{\mathcal{S}}$ lifts to a one-form field in four dimensions under M-/F-theory duality $[1,2]$. The geometric realisation and the physics of extra sections has been studied extensively in the recent literature [4-22].

\subsection{Torsional sections and divisor classes}

Let us now consider the divisor class $R$ of a torsional meromorphic section of order $k$ such that $R-Z$ is a generator of the torsional part of the Mordell-Weil group of $\hat{Y}_{4}$. Combining

\footnotetext{
${ }^{4}$ By contrast, massive U(1)s in F-theory can be understood along the lines of $[4,51-53]$; see also [54, 55] for a similar mechanism at work in a different context.
} 
the theory outlined in section 2.2 with the properties of the Shioda map one can conclude that there exists now a fractional linear combination of resolution divisors $F_{i}$ such that

$$
\Sigma:=R-Z-\pi^{-1}(\delta)+\frac{1}{k} \sum a_{i} F_{i} \quad \text { with } \quad a_{i} \in \mathbb{Z}
$$

is trivial in $N S\left(\hat{Y}_{4}\right) \otimes \mathbb{Q}$ and thus in particular in $H^{2}\left(\hat{Y}_{4}, \mathbb{R}\right)$. Indeed, as described in section 2.2 , it is guaranteed that $R-Z$ can be expressed as a linear combination with $\mathbb{Q}$ coefficients of the generators of $\mathcal{T}$, the subgroup of $N S\left(\hat{Y}_{4}\right)$ generated by $\left[\sigma_{0}\right]$, the resolution divisors $F_{i}$ and $\pi^{-1}(\delta)$ for some divisor class $\delta$ on $\mathcal{B}$. Thus, $R-Z$ minus this linear combination is trivial in $N S\left(\hat{Y}_{4}\right) \otimes \mathbb{Q}$. On the other hand, the Shioda map gives a specific such linear combination of the form (3.3) as

$$
\varphi(R-Z)=R-Z-\pi^{-1}(\delta)+\sum_{i} l_{i} F_{i} .
$$

The rational numbers $l_{i}$ are in fact of the form $\frac{a_{i}}{k}$ with $a_{i} \in \mathbb{Z}$. Since $\varphi$ is a homomorphism, $\varphi(k(R-Z))=k\left(R-Z-\pi^{-1}(\delta)+\sum_{i} l_{i} F_{i}\right)$ and this must be trivial in $N S\left(\hat{Y}_{4}\right) \otimes \mathbb{Q}$ because $R-Z$ is $k$-torsion. Furthermore, since $N S\left(\hat{Y}_{4}\right) \otimes \mathbb{Q}$ is torsion-free, this implies that $R-Z-\pi^{-1}(\delta)+\sum_{i} l_{i} F_{i}$ is trivial in $N S\left(\hat{Y}_{4}\right) \otimes \mathbb{Q}$, as claimed above.

We will exemplify this general fact for situations in which $\hat{Y}_{4}$ is a hypersurface in a toric ambient space. In our examples, $-k \Sigma$ turns out to be a toric divisor on the toric ambient space which does not intersect the Calabi-Yau hypersurface $\hat{Y}_{4}$. Furthermore, in the toric examples we will consider the base divisor $\delta$ will be given by $\overline{\mathcal{K}}_{\mathcal{B}}$, the anti-canonical divisor of $\mathcal{B} .^{5}$

Since $[\Sigma]$ is trivial as an element of $H^{2}\left(\hat{Y}_{4}, \mathbb{R}\right)$, it does not give rise to an extra $\mathrm{U}(1)$ factor as would be the case if $R$ were a non-torsional rational section. We may use the triviality of $\Sigma$ in $N S\left(\hat{Y}_{4}\right) \otimes \mathbb{Q}$ to write

$$
\Xi_{k} \equiv R-Z-\pi^{-1}(\delta)=-\frac{1}{k} \sum_{i} a_{i} F_{i}, \quad a_{i} \in \mathbb{Z},
$$

which by construction defines an element in $H^{2}\left(\hat{Y}_{4}, \mathbb{Z}\right)$. One may be forgiven for thinking that the existence of a $k$-torsional point on the elliptic fiber induces a $k$-torsional element in $H^{2}\left(\hat{Y}_{4}, \mathbb{Z}\right)$. This is almost true but misses possible complications in the degenerate fibers at codimension-one singular loci whose resolution introduces the extra divisor classes $F_{i}$. Indeed, from (3.7) we see that while the class $\left[\Xi_{k}\right]$ is not torsion in the cohomology $H_{\mathbb{Z}}^{1,1}\left(\hat{Y}_{4}\right)=H^{2}\left(\hat{Y}_{4}, \mathbb{Z}\right) \cap H^{1,1}\left(\hat{Y}_{4}\right)$, it does represent a $k$-torsional element in the quotient cohomology $H_{\mathbb{Z}}^{1,1}\left(\hat{Y}_{4}\right) /\left\langle\left[F_{i}\right]\right\rangle_{\mathbb{Z}}$ of classes modulo integer linear combinations of resolution classes. Namely,

$$
k \cdot\left[\Xi_{k}\right]=-\sum_{i} a_{i}\left[F_{i}\right]=0 \bmod f \in\left\langle\left[F_{i}\right]\right\rangle_{\mathbb{Z}}
$$

which establishes $\left[\Xi_{k}\right]$ as $k$-torsion up to resolution divisors. We will give an intuitive explanation for the appearance of such a torsional element from the geometry of the elliptic fibration in the examples below - see section 4.1.2.

\footnotetext{
${ }^{5}$ This is due to the fact that we are only considering fibrations which are blow-ups of the Weierstrass type or have at least one holomorphic section.
} 


\subsection{The global structure of the gauge group in presence of Mordell-Weil tor- sion}

While, as described, the existence of a torsional section does not give rise to any new U(1) groups, it does have profound consequences on the physical properties of the F-theory compactification by restricting the matter spectrum and, equivalently, the global structure of the gauge group.

In F-theory, the non-abelian gauge algebra $\mathfrak{g}$ is dictated entirely by the singularity structure of the elliptic fibration $\hat{Y}_{4}$ in codimension one. The resolution divisors $F_{i}$ correspond to the generators of the Cartan subalgebra $\mathfrak{h}$ of $\mathfrak{g}$. The Cartan generators, or equivalently the resolution divisors, span the coroot lattice $Q^{\vee}=\left\langle F_{i}\right\rangle_{\mathbb{Z}}$. On the other hand, the information about the global structure of the non-abelian gauge group $G$ with Lie algebra $\mathfrak{g}$ is reflected in the representation content. In F-theory localised charged massless matter states in representation $\rho$ of the full gauge group $G$ arise from M2-branes wrapping suitable fiber components $\mathbb{P}_{\rho}^{1}$ over codimension-two loci on $\mathcal{B}$ corresponding to the intersection of several components of the discriminant locus, or to self-intersections of its components. The fiber components in question can be identified with the weights of the representation $\rho$. The weights of all representations which are realized in the geometry span the weight lattice $\Lambda$. The coweight lattice $\Lambda^{\vee}$ is the dual lattice, defined by the integer pairing with the weight lattice $\Lambda$,

$$
\Lambda^{\vee} \times \Lambda \rightarrow \mathbb{Z}
$$

Geometrically, the coroot lattice $Q^{\vee} \subseteq \Lambda^{\vee}$ is spanned by the resolution divisors $F_{i}$, and the pairing is the intersection with the fiber components $\mathbb{P}_{\rho}^{1}$ associated with the matter representations.

The relation between the representation data and the global structure of the gauge group be understood as follows: For definiteness consider a semi-simple Lie group $G$. For such $G$ recall, e.g. from $[56,57]$, that

$$
\pi_{1}(G) \approx \frac{\Lambda^{\vee}}{Q^{\vee}}
$$

It will be useful to compare $G$ to its universal cover $G_{0}$, which has the same Lie algebra $\mathfrak{g}$ and whose coweight lattice is by definition $\Lambda_{0}^{\vee}=\left\langle F_{i}\right\rangle_{\mathbb{Z}}$. The dual weight lattice $\Lambda_{0}$ then contains all information about the representations that occur in a gauge theory with gauge group $G_{0}$. Since by assumption $\Lambda_{0}^{\vee}=Q^{\vee}$, the group $G_{0}$ is simply-connected.

Now, for definiteness suppose that the F-theory compactification gives rise to gauge algebra $\mathfrak{g} \oplus \mathfrak{g}^{\prime}$, where $\mathfrak{g}$ and $\mathfrak{g}^{\prime}$ are both semi-simple and whose Cartan subgroups are spanned by two sets of resolution divisors $F_{i}$ and $F_{i}^{\prime}$. The gauge algebra $\mathfrak{g}^{\prime}$ and its gauge group $G^{\prime}$ will be mere spectators in what follows, but we include them to be more general. We are interested in the structure of the global gauge group $G \times G^{\prime}$. Suppose furthermore that the Mordell-Weil group has $k$-torsion and that the class $\Xi_{k}$ defined in (3.7) involves only the Cartan generators $F_{i}$ of $\mathfrak{g}$, but not the generators $F_{i}^{\prime}$ of $\mathfrak{g}^{\prime}$. The class $\Xi_{k}$ is integer and therefore its intersection with the split fiber components $\mathbb{P}_{\rho}^{1}$ is integer as well. Group theoretically this implies that we can identify $\Xi_{k}$ with a coweight of $G$. Having fractional 


\begin{tabular}{|c|c|}
\hline Lie algebra & Center of universal covering group \\
\hline$A_{n \geq 1}$ & $\mathbb{Z}_{n+1}$ \\
$B_{n \geq 2}$ & $\mathbb{Z}_{2}$ \\
$C_{n \geq 3}$ & $\mathbb{Z}_{2}$ \\
$D_{2 n+1 \geq 4}$ & $\mathbb{Z}_{4}$ \\
$D_{2 n \geq 4}$ & $\mathbb{Z}_{2} \oplus \mathbb{Z}_{2}$ \\
$E_{6}$ & $\mathbb{Z}_{3}$ \\
$E_{7}$ & $\mathbb{Z}_{2}$ \\
$E_{8}$ & - \\
$F_{4}$ & - \\
$G_{2}$ & - \\
\hline
\end{tabular}

Table 1. Simple Lie algebras and the center of their universal covering groups.

coefficients in $\frac{1}{k} \mathbb{Z}$ with respect to the $F_{i}$, the class $\Xi_{k}$ corresponds to a coweight in a coweight lattice $\Lambda^{\vee}$ which is finer (by order $k$ ) compared to the sublattice $\Lambda_{0}^{\vee}=\left\langle F_{i}\right\rangle_{\mathbb{Z}}$ spanned by the $F_{i}$ alone. Therefore $\pi_{1}(G) \approx \frac{\Lambda^{\vee}}{Q^{\vee}}$ acquires a $\mathbb{Z}_{k}$ component compared to the first fundamental group of $G_{0}$. This leads to non-simply connected gauge groups. Since the universal covering group $G_{0}$ is simply connected, the gauge group $G \times G^{\prime}$ in such an F-theory compactification with Mordell-Weil torsion $\mathbb{Z}_{k}$ has in fact first fundamental group

$$
\pi_{1}(G) \times \pi_{1}\left(G^{\prime}\right)=\mathbb{Z}_{k} \times \pi_{1}\left(G^{\prime}\right),
$$

where the spectator group $G^{\prime}$ is unaffected by the Mordell-Weil torsion.

At the same time, the integer pairing (3.9) of coweights and weights forces the weight lattice $\Lambda$ to be coarser compared to the weight lattice $\Lambda_{0}$ dual to $\Lambda_{0}^{\vee}$, and the weights realized in the geometry become a subset of all weights that would be possible on the basis of the Lie algebra alone. Not only can one verify, as is clear by construction, that the geometrically realised representations all have integer pairing with the coweight $-\frac{1}{k} \sum_{i} a_{i} F_{i}$ (appearing on the right of (3.7)), but also other representations which would be present in more generic fibrations without torsional sections have only fractional such pairing and are therefore 'forbidden'.

Equivalently, we can think of Mordell-Weil torsion as affecting the center $Z_{G}$ of the gauge group $G$. The center $Z_{G}$ of a semi-simple Lie group $G$ is given by $[56,57]$

$$
Z_{G} \approx \frac{\Lambda}{Q}
$$

where $Q \subset \Lambda$ the root lattice (see figure 1 for a list of the center of the universal covering groups of the simple Lie algebras). Geometrically $Q$ is spanned by the fiber components associated with the adjoint representation of $G$ localised in codimension one. As a reference consider again the universal cover group $G_{0}$ introduced above with center $Z_{G_{0}}$. Since 
Mordell-Weil torsion $\mathbb{Z}_{k}$ renders $\Lambda$ coarser by a factor of $\mathbb{Z}_{k}$ compared to $\Lambda_{0}$, the center of $G$ is smaller by the same amount,

$$
Z_{G}=Z_{G_{0}} / \mathbb{Z}_{k}
$$

Note that this requires that $\mathbb{Z}_{k}$ be a subgroup of the center of $G_{0}$, which constrains the possible gauge algebra $\mathfrak{g}$ that can possibly appear. By contrast, any extra spectator Lie algebra $\mathfrak{g}^{\prime}$ whose generators do not enter $\Xi_{k}$ is unconstrained. For example, if the MordellWeil torsion is $\mathbb{Z}_{2}$, then a gauge algebra $\mathfrak{g}=\mathfrak{s u}(k)$ is possible only for $k=2 n$ - see the discussion in section 4.5 for an explicit construction. Furthermore, the total gauge group is given by

$$
G_{0} / \mathbb{Z}_{k} \times G^{\prime}
$$

This can be directly understood in terms of the construction of our coweight element $\Xi_{k}$ in (3.7). Exponentiation of $\Xi_{k}$ generates a $\mathbb{Z}_{k}$ subgroup of $Z_{G_{0}}$. Since $\Xi_{k}$ has integer pairing with every representation that is present (i.e. with every lattice point in the weight lattice $\Lambda$, but not not $\Lambda_{0}$ ), the corresponding center element (viewed as an element of $G_{0}$ ) acts trivially on every such representation; the actual gauge group is therefore not $G_{0} \times G^{\prime}$, but $G_{0} / \mathbb{Z}_{k} \times G^{\prime}$.

Indeed, to construct an element in the center of $G_{0}$ one exponentiates a linear combination $\Xi=\sum m_{i} F_{i}$ of Cartan generators $F_{i}$ for suitable coefficients $m_{i}$. We denote by $\rho_{d}$ a $d$-dimensional representation of $\mathfrak{g}$. A state $\left|\lambda^{n}, \rho_{d}\right\rangle$ in the representation $\rho_{d}$ is labeled by the weight $\lambda^{n}$ in the weight system of $\rho_{d}$. Letting $\Xi$ act on such a state gives

$$
\Xi \cdot\left|\lambda^{n}, \rho_{d}\right\rangle=\sum_{i} m_{i} \lambda_{i}^{n}\left|\lambda^{n}, \rho_{d}\right\rangle, \quad n=1, \ldots, d, \quad i=1, \ldots, r,
$$

where $\lambda_{i}^{n}$ is the eigenvalue of $F_{i}$ on this state vector. An element $c$ in the center $Z_{G_{0}} \in G_{0}$ commutes with any element in $G_{0}$ and is represented as a multiple of the $d \times d$ unit matrix when acting on the state $\left|\lambda^{n}, \rho_{d}\right\rangle$, i.e

$$
c \rho_{\rho_{d}} \cdot\left|\lambda^{n}, \rho_{d}\right\rangle=a_{n} \mathbb{1} \cdot\left|\lambda^{n}, \rho_{d}\right\rangle
$$

for $a_{n} \in \mathbb{C}$. To identify $c$ as the exponentiation of $\Xi$ we identify

$$
a_{n}=\exp \left(2 \pi i \sum m_{i} \lambda_{i}^{n}\right)
$$

For $c$ to lie in a $\mathbb{Z}_{k}$ subgroup of the center of $G_{0}, c^{k}$ acts as $\mathbb{1}$ on any representation $\rho_{d}$, or equivalently $\left(a_{n}\right)^{k}=1$ for all $n$. Therefore, if we identify $\Xi$ with the $k$-fractional linear combination $\Xi_{k}=-\frac{1}{k} \sum_{i} a_{i} F_{i}$, we see that this does indeed generate a $\mathbb{Z}_{k}$ subgroup of $Z_{G_{0}}$. Moreover, since $\Xi_{k}$ has integer pairing with all weights in the weight lattice $\Lambda$ of the actual gauge group $G$, the element $c$ acts trivially on every such representation. We can therefore view $G$ as the result of 'gauging' $\mathbb{Z}_{k}$, i.e. $G=G_{0} / \mathbb{Z}_{k}$, as claimed. Finally, note that all results of this section generalize to more complicated Mordell-Weil torsion groups of the form $\mathbb{Z}_{k_{1}} \oplus \ldots \oplus \mathbb{Z}_{k_{n}}$. 


\section{Mordell-Weil group $\mathbb{Z}_{2}$}

In the subsequent sections we exemplify the structure of F-theory compactifications on elliptic fibrations with torsional Mordell-Weil group as outlined above. Ref. [32] has derived the defining equations describing elliptic fibrations with Mordell-Weil group $\mathbb{Z}_{k}$ for $k=$ $2,3,4,5,6, \mathbb{Z}_{2} \oplus \mathbb{Z}_{n}$ with $n=2,4$ and $\mathbb{Z}_{3} \oplus \mathbb{Z}_{3}$ as hypersurfaces in $\mathbb{P}_{2,3,1}[6]$ fibrations. As it turns out, the restriction of the complex structure moduli of the fibration necessary for the Mordell-Weil group to have torsion induces singularities in the fiber over divisors on the base $\mathcal{B}$. To explicitly analyse these singular loci and their resolution we focus in this work on the subset of geometries in the list of [32] which can be treated torically as certain hypersurfaces. As noted already, there exist 16 reflexive polygons in two dimensions which describe an elliptic curve as a hypersurface in a toric ambient space. Of these only three admit torsional sections in the Mordell-Weil group as the intersection of a toric divisor with the generic hypersurface defined by the dual polygon. The Mordell-Weil group of these fibrations has already been provided in [15]. As we will show, they correspond to the geometries with Mordell-Weil group $\mathbb{Z}_{2}$ and $\mathbb{Z}_{3}$ as well as a further specialisation of the $\mathbb{Z}_{2}$-model in the list of [32]. For each of these three fibration types we construct a compact model fibered over a generic base $\mathcal{B}$ and analyse in detail the interplay between the torsional sections and the global structure of the gauge group. In addition we implement further non-abelian gauge symmetries by the construction of toric tops [43].

\subsection{An $\mathrm{SU}(2) / \mathbb{Z}_{2}$-fibration}

We begin with the simplest example of an elliptic fibration with torsional Mordell-Weil group, which turns out to be $\mathbb{Z}_{2}$. As derived in [32], an elliptic fibration with a $\mathbb{Z}_{2}$-torsional section admits a representation as the hypersurface $P=0$ with

$$
P=-y^{2}-a_{1} x y z+x^{3}+a_{2} x^{2} z^{2}+a_{4} x z^{4}
$$

and $[x: y: z]$ fiber coordinates in a $\mathbb{P}_{2,3,1}$-fibration over some base $\mathcal{B}$. To ensure that the variety $P=0$ satisfies the Calabi-Yau condition the coefficients $a_{i}$ must be sections of $\overline{\mathcal{K}}_{\mathcal{B}}^{i}$ with $\overline{\mathcal{K}}_{\mathcal{B}}$ the anti-canonical bundle of the base $\mathcal{B}$. Note that (4.1) corresponds to an otherwise generic Tate model with $a_{6} \equiv 0$ and $a_{3} \equiv 0$. It can therefore be viewed as a further specialisation of the $\mathrm{U}(1)$ restricted Tate model, defined in [4] by setting $a_{6} \equiv 0$. The latter has Mordell-Weil group $\mathbb{Z}$ and in turn represents a special case of the elliptic fibrations with Mordell-Weil group $\mathbb{Z}$ as described in [9].

\subsubsection{Singularity structure and resolution}

The elliptic fibration (4.1) is easily brought into Weierstrass form (2.1) with

$$
f=a_{4}-\frac{1}{3}\left(a_{2}+\frac{a_{1}^{2}}{4}\right)^{2}, \quad g=\frac{1}{27}\left(a_{2}+\frac{a_{1}^{2}}{4}\right)\left(2\left(a_{2}+\frac{a_{1}^{2}}{4}\right)^{2}-9 a_{4}\right) .
$$

From $f$ and $g$ and the discriminant

$$
\Delta=\frac{1}{16} a_{4}^{2}\left(4 a_{4}-\left(a_{2}+\frac{1}{4} a_{1}^{2}\right)^{2}\right)
$$


one infers an $\mathfrak{s u}(2)$-singularity at $a_{4}=0$. Indeed, the gradient of (4.1) in the patch $z \neq 0$,

$$
d P=\left(-a_{1} y+3 x^{2}+2 a_{2} x+a_{4}\right) d x-\left(2 y+a_{1} x\right) d y-x y d_{B} a_{1}+x^{2} d_{B} a_{2}+x d_{B} a_{4},
$$

with $d_{B}$ the total derivative with respect to the base coordinates, vanishes together with the hypersurface equation (4.1) for $x=y=a_{4}=0$. The situation is similar to the U(1)restricted model with $a_{6} \equiv 0$ but $a_{3} \neq 0$ [4], in which, however, the singularity appeared over the curve $\left\{a_{3}=0\right\} \cap\left\{a_{4}=0\right\}$ on $\mathcal{B}$. Since in (4.1) $a_{3}$ is set to zero from the very beginning, the $\mathfrak{s u}(2)$ locus is promoted to the divisor $\left\{a_{4}=0\right\}$. We will come back to this enhancement of the $\mathfrak{u}(1)$ gauge algebra of the $\mathrm{U}(1)$ restricted Tate model to $\mathfrak{s u}(2)$ by setting $a_{3} \equiv 0$ in section 5.2.

To resolve the singularity we perform a blow-up in the fiber ambient space

$$
x \rightarrow s x, \quad y \rightarrow s y .
$$

Since $a_{6} \equiv 0$, this does not spoil the Calabi-Yau condition of the hypersurface as one can see from the proper transform of (4.1) given by

$$
\hat{P}=-y^{2} s-a_{1} x y z s+x^{3} s^{2}+a_{2} x^{2} z^{2} s+a_{4} x z^{4},
$$

which is checked to be smooth (see $[4,6]$ for the analogous blow-up if $a_{3} \neq 0$ ). In order to facilitate the description of the $\mathbb{Z}_{2}$-torsional section it turns out useful to perform a further ambient space blow-up

$$
s \rightarrow t s, \quad x \rightarrow t x,
$$

under which the proper transform of (4.5) becomes

$$
\hat{P}=-y^{2} s-a_{1} x y z s t+x^{3} s^{2} t^{4}+a_{2} x^{2} z^{2} s t^{2}+a_{4} x z^{4} .
$$

The Stanley-Reisner ideal relations after the two blow-ups are

$$
\text { SR-i : }\{y t, y x, s x, s z, t z\},
$$

and we observe that the divisor $X:\{x=0\}$ does not intersect the hypersurface. Hence $x$ can be set to one in (4.7) and from now on we will analyse the fibration $\hat{P}=0$ with

$$
\hat{P}=-y^{2} s-a_{1} y z s t+s^{2} t^{4}+a_{2} z^{2} s t^{2}+a_{4} z^{4}
$$

over a suitable base $\mathcal{B}$. If $\mathcal{B}$ is 3 -dimensional, this defines an elliptically fibered Calabi-Yau 4 -fold $\hat{Y}_{4}$. The weight matrix of the homogeneous coordinates can be taken to be

\begin{tabular}{|c|c|c|c|c|}
\hline$y$ & $z$ & $s$ & $t$ & $\sum$ \\
\hline 2 & 1 & 0 & 1 & 4 \\
\hline 1 & 1 & 2 & 0 & 4 \\
\hline
\end{tabular}

and the Stanley-Reisner ideal simplifies to

$$
\{y t, s z\} \text {. }
$$



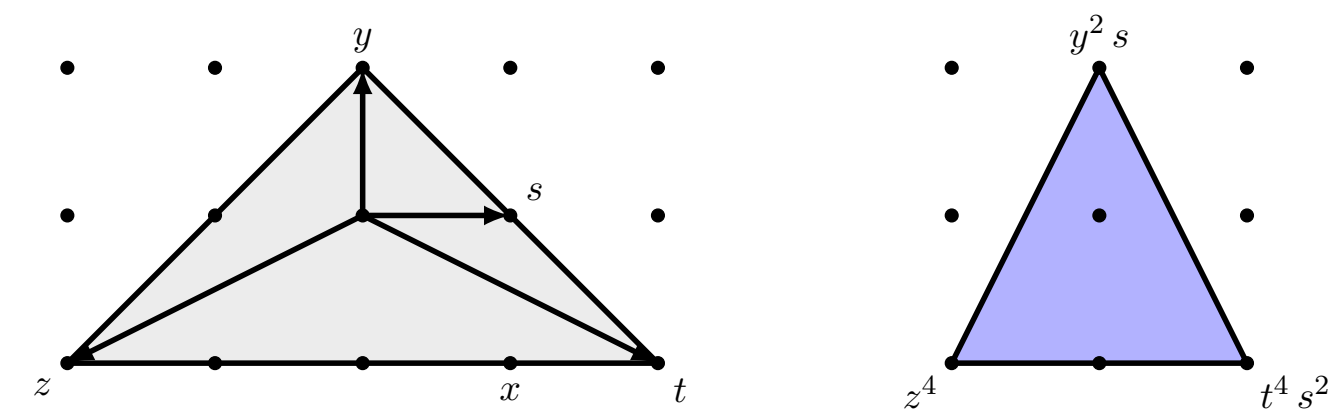

Figure 1. Polygon 13 of [43] together with its dual polygon. The coordinate $x$ is blown-down, and not part of the fan.

Note that the weight matrix (4.10) coincides with the weight matrix as read off from the toric fan depicted in figure 1, which corresponds to polygon 13 in the list [43] of 16 torically embedded hypersurface elliptic curves. The fibration (4.9) with $s \equiv 1$, corresponding to the blow-down of the resolution divisor associated with the $\mathfrak{s u}(2)$ singularity over $a_{4}=0$, has been analysed previously in [41] and shown to correspond to an elliptic fibration with restricted $\mathrm{SL}(2, \mathbb{Z})$ monodromy. We will analyze this relation in more detail in section 4.5.

The advantage of passing to the hypersurface representation (4.9) is that the $\mathbb{Z}_{2^{-}}$ torsional point on the elliptic fiber is now explictly given by the intersection of the fiber with the toric divisor

$$
T: t=0
$$

This can be checked via the group law on the elliptic curve. We will henceforth denote $T$ as the $\mathbb{Z}_{2}$ section of the fibration. The holomorphic zero-section is given by $Z: z=0$.

To study the geometry further we note that the fibration restricted to the $\mathfrak{s u}(2)$ sublocus $\left\{a_{4}=0\right\}$ in the discriminant (4.2) factorises as

$$
\left.\hat{P}\right|_{a_{4}=0}=s\left(-y^{2}-a_{1} y z t+\left(s t^{4}+a_{2} z^{2} t^{2}\right)\right) .
$$

The resolution divisor $S: s=0$ is a $\mathbb{P}^{1}$-fibration over the locus $\left\{a_{4}=0\right\}$ on $\mathcal{B}$ as the coordinate $s$ is just a toric ambient space coordinate. The other irreducible component of (4.13) is quadratic in $y$ and must therefore be studied in more detail. Note first that this component does not intersect the $\mathbb{Z}_{2}$ section $T$, but only the holomorphic zero-section $Z$. Since $z$ and $t$ cannot both vanish along it, we can go to the patch where $y$ and $s$ can vanish simultaneously. Here the second factor of (4.13) becomes

$$
y^{2}+a_{1} y-\left(s+a_{2}\right)=0 .
$$

The discriminant of this quadratic equation is a linear function in $s$ so that we find one branching point in the $s$-plane. Since the point at ' $s=\infty$ ' $(z=0)$ is also single valued, we can take the branch-cut from $s=-\left(\frac{1}{4} a_{1}^{2}+a_{2}\right)$ to infinity. Gluing the two $\mathbb{P}^{1} \mathrm{~s}$ viewed as compactified complex planes along the branch-cut, we obtain again a $\mathbb{P}^{1}$. The two irreducible parts of (4.13) intersect each other in two points, as can be seen from (4.14). The factorised fiber over the base divisor $\left\{a_{4}=0\right\}$ is depicted on the left in figure 2. Over 

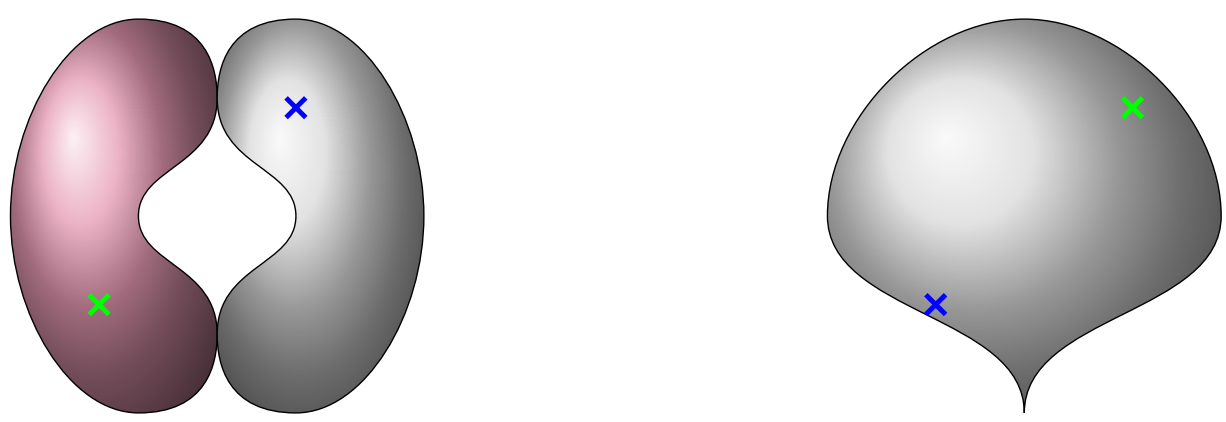

Figure 2. To the left we depict the factorised fiber over the base locus $a_{4}=0$; the purple $\mathbb{P}^{1}$ indicates the $s=0$ part while the grey $\mathbb{P}^{1}$ is the second irreducible part of the elliptic curve. To the right the fiber over the base locus $a_{4}=\frac{1}{4}\left(a_{2}+\frac{1}{4} a_{1}^{2}\right)^{2}$ is shown. The multiplicity is one, and the fiber is singular. The blue and green crosses indicate the specified points $z=0$ and the $\mathbb{Z}_{2}$-point $t=0$ of the elliptic curve, respectively.

the zero set of the second factor of the discriminant (4.2),

$$
4 a_{4}-\left(a_{2}+\frac{1}{4} a_{1}^{2}\right)^{2}=0,
$$

we analyse the fiber structure by substituting (4.15) into (4.9). This gives the hypersurface equation

$$
\left.\hat{P}\right|_{(\ldots=0)}=-y^{2} s-a_{1} y z s t+s^{2} t^{4}+a_{2} z^{2} s t^{2}+\frac{1}{4}\left(a_{2}+\frac{1}{4} a_{1}^{2}\right)^{2} z^{4} .
$$

To determine the fiber type, we can go to the patch where $y$ and $z$ are allowed to vanish simultaneously. We set $s=1$ since the divisor $\{s=0\}$ does not intersect the elliptic curve away from $\left\{a_{4}=0\right\}$ and complete the square as

$$
\begin{aligned}
& y^{2}+a_{1} y z=1+a_{2} z^{2}+\frac{1}{4}\left(a_{2}+\frac{1}{4} a_{1}^{2}\right)^{2} z^{4} \\
& \Rightarrow\left(y+\frac{1}{2} a_{1} z\right)^{2}=1+\left(a_{2}+\frac{1}{4} a_{1}^{2}\right) z^{2}+\frac{1}{4}\left(a_{2}+a_{1}^{2}\right)^{2} z^{4} \\
& \Rightarrow\left(y+\frac{1}{2} a_{1} z\right)^{2}=\left(1+\frac{1}{2}\left(a_{2}+\frac{1}{4} a_{1}^{2}\right) z^{2}\right)^{2} \\
& \Rightarrow\left(y+\frac{1}{2} a_{1} z-1-\frac{1}{2}\left(a_{2}+\frac{1}{4} a_{1}^{2}\right) z^{2}\right)\left(y+\frac{1}{2} a_{1} z+1+\frac{1}{2}\left(a_{2}+\frac{1}{4} a_{1}^{2}\right) z^{2}\right)=0 .
\end{aligned}
$$

Therefore, it appears as if the elliptic curve factorises into two rational curves. However, these two $\mathbb{P}^{1} \mathrm{~s}$ are equivalent as follows from the second row of the weight matrix (4.10) because the equivalence relation $(y, z) \sim(-y,-z)$ is left over after setting $s$ to one. ${ }^{6}$ Thus the fiber is just a single rational curve; moreover, it has a singular point, cf. figure 2, at $y=-\frac{1}{2} a_{1} z, s=-\frac{1}{8}\left(a_{1}^{2}+4 a_{2}\right) z^{2}$ (and $t=1$ due to the Stanley-Reisner ideal), where the

\footnotetext{
${ }^{6}$ This can also be seen from the $N$-lattice polygon of figure 1 because $y$ and $z$ do not span the lattice. The patch where $y$ and $z$ are allowed to vanish simultaneously is, therefore, $\mathbb{C}^{2} / \mathbb{Z}_{2}$ and not $\mathbb{C}^{2}$ as one would naïvely think.
} 
gradient along the fiber coordinates vanishes even though the fibration as such is nonsingular. Thus the fiber is of Kodaira-type $I_{1}$, and the locus (4.15) does not give rise to any further gauge symmetry.

Interestingly, apart from the codimension-one splitting of the fiber over $\left\{a_{4}=0\right\}$ no further degeneration of the fiber occurs in higher codimension. In particular, the fiber over the intersection curve $\left\{a_{4}=0\right\} \cap\left\{a_{2}+\frac{1}{4} a_{1}^{2}=0\right\}$ of the two components of the discriminant does not factorise further. This can be understood by considering the vanishing of $f$ and $g$ along that locus: $f$ vanishes to order $1, g$ vanishes to order 2 and the discriminant $\Delta$ consequently to order 3 , giving a Kodaira fiber of type $I I I$. This type of fiber has two components just like the familiar $A_{1}$-fiber, but they are tangent to each other rather than meeting at two distinct points, and there is no enhancement or matter (consistent with [58, 59]). This is remarkable because naively one might have expected an enhancement from $A_{1}$ to $A_{2}$ at the intersection of the $A_{1}$-locus with the $I_{1}$-component of the discriminant and thus localised massless matter in the fundamental of $\mathfrak{s u}(2)$. The absence of this enhancement and the associated fundamental representation is a typical property of fibrations with torsional Mordell-Weil group. To summarize, the fibration (4.1) gives rise to an F-theory compactification with gauge algebra $\mathfrak{s u}(2)$ and no localised charged matter.

\subsubsection{Torsional divisors and free quotient}

The absence of charged localized matter in the fundamental representation is a consequence of the $\mathbb{Z}_{2}$ Mordell-Weil group and the resulting global structure of the gauge group. To see this let us first exemplify how the torsional Mordell-Weil group of the elliptic fiber induces a torsional element in $H^{1,1}\left(\hat{Y}_{4}, \mathbb{Z}\right)$ modulo the integer lattice spanned by the resolution divisors. In the present model with gauge algebra $\mathfrak{g}=\mathfrak{s u}(2)$ the lattice of resolution divisors is simply $\langle S\rangle_{\mathbb{Z}}$. To find the element $\Sigma_{2}$ of the form (3.5) we make an Ansatz and demand that (3.4) be satisfied. In the present situation this amounts to demanding that $\Sigma_{2}$ have 'one leg in the fiber' and that it be orthogonal to the exceptional divisor $S$, in the sense that for all $\omega_{4} \in H^{4}(B)$ and $\omega_{2} \in H^{2}(B)$

$$
\int_{\hat{Y}_{4}} \Sigma_{2} \wedge Z \wedge \pi^{*} \omega_{4}=\int_{\hat{Y}_{4}} \Sigma_{2} \wedge \pi^{*} \omega_{2} \wedge \pi^{*} \omega_{4}=\int_{\hat{Y}_{4}} \Sigma_{2} \wedge S \wedge \pi^{*} \omega_{4}=0 .
$$

This uniquely determines

$$
\Sigma_{2}=T-Z-\overline{\mathcal{K}}+\frac{1}{2} S
$$

with $\overline{\mathcal{K}}=\pi^{-1} \overline{\mathcal{K}}_{\mathcal{B}}$. This element is in fact trivial in $H^{2}\left(\hat{Y}_{4}, \mathbb{R}\right)$. Indeed, recall that the fibration $\hat{Y}_{4}$ is described as the hypersurface (4.7) in an ambient toric space. Consider the toric divisor $X:\{x=0\}$ in this ambient space. Its class is

$$
X=2 Z-S-2 T+2 \overline{\mathcal{K}}=-2 \Sigma_{2} .
$$

However, as discussed, $X$ does not intersect the hypersurface $\hat{Y}_{4}$ and therefore its class is trivial on the hypersurface. Thus also $\Sigma_{2}$ is trivial in $H^{1,1}\left(\hat{Y}_{4}, \mathbb{R}\right)$. This implies that

$$
\Xi_{2}:=T-Z-\overline{\mathcal{K}}=-\frac{1}{2} S,
$$

thereby identifying $\Xi_{2}$ as 2-torsion in $H^{1,1}\left(\hat{Y}_{4}, \mathbb{Z}\right) /\langle S\rangle_{\mathbb{Z}}$. 
According to the discussion in section 3.2 , associated with $\Xi_{2}$ is an extra coweight defined over $\frac{1}{2} \mathbb{Z}$. Thus, to preserve the pairing with the weights, the weight lattice is forced to be coarser. In particular the representation 2 of $\mathfrak{s u}(2)$ cannot be present in this model as its weight would have half-integer pairing with the fractional coweight $\Xi_{2}=-\frac{1}{2} S$, in contradiction with the fact that $T-Z-\overline{\mathcal{K}}$ is manifestly integer. This is the deeper reason behind the absence of a fundamental representation at the intersection of the $\mathfrak{s u}(2)$-divisor $\left\{a_{4}=0\right\}$ with the second discriminant component. The gauge group of the model is thus

$$
G=\mathrm{SU}(2) / \mathbb{Z}_{2}
$$

with $\pi_{1}(G)=\mathbb{Z}_{2}$.

One can give an intuitive geometric explanation for the appearance of the 2-torsion element $\Xi_{2}$ in $H^{1,1}\left(\hat{Y}_{4}, \mathbb{Z}\right) /\langle S\rangle_{\mathbb{Z}}$ as follows: Restrict the elliptically fibered Calabi-Yau $\hat{Y}_{4}$ over $\mathcal{B}$ given by the hypersurface equation (4.7) to $\mathcal{B} \backslash\left\{a_{4}=0\right\}$. As will be discussed momentarily, the resulting space $\hat{Y}_{4}^{\prime}$ is a free $\mathbb{Z}_{2}$ quotient,

$$
\hat{Y}_{4}^{\prime}=\widetilde{\hat{Y}_{4}^{\prime}} / \mathbb{Z}_{2}
$$

with $\widetilde{\hat{Y}_{4}^{\prime}}$ an elliptic fibration over $\mathcal{B} \backslash\left\{a_{4}=0\right\}$. Correspondingly

$$
\pi_{1}\left(\hat{Y}_{4}^{\prime}\right) \supset \mathbb{Z}_{2}
$$

where additional discrete torsion pieces may arise if $\pi_{1}\left(\mathcal{B} \backslash\left\{a_{4}=0\right\}\right)$ is non-trivial. Since the resolution divisor $S$ is fibered over $\left\{a_{4}=0\right\}$ this is in agreement with the appearance of a torsional element in $H^{1,1}\left(\hat{Y}_{4}, \mathbb{Z}\right) /\langle S\rangle_{\mathbb{Z}}$.

The relation (4.21) can be seen as follows: consider the fibration over a generic locus on the base $\mathcal{B}$ where $a_{4} \neq 0$. Since the resolution divisor $s=0$ intersects the fiber only over $\left\{a_{4}=0\right\}$ we can set $s$ to one away from that locus. Then (4.9) becomes

$$
y^{2}+a_{1} y z t=t^{4}+a_{2} z^{2} t^{2}+a_{4} z^{4} .
$$

This is a special $\mathbb{P}_{1,1,2}[4]$ fibration with homogeneous coordinates $[t: z: y]$, which in addition to the equivalence relation $(t, z, y) \sim\left(\lambda t, \lambda z, \lambda^{2} y\right)$ enjoys a further $\mathbb{Z}_{2}$ identification

$$
t \sim-t, \quad y \sim-y .
$$

In fact, the most generic $\mathbb{P}_{1,1,2}[4]$ representation of an elliptic curve contains the nine terms

$$
y^{2}, t^{4}, z^{4}, z^{2} t^{2}, y z t ; \quad y t^{2}, y z^{2}, z t^{3}, t z^{3} .
$$

Precisely the first five terms present in (4.23) are compatible with the $\mathbb{Z}_{2}$ identification (4.24). Note that by a coordinate redefinition we can set $a_{1} \equiv 0$, thereby arriving at the special $\mathbb{P}_{1,1,2}[4]$-fibration that goes by the name of the Légendre family. In any case, we can view (4.23) as the result of starting with a $\mathbb{P}_{1,1,2}[4]$ fibration described by the hypersurface equation

$$
y^{2}+a_{1} y z t=t^{4}+a_{2} z^{2} t^{2}+a_{4} z^{4}+c_{1} y t^{2}+c_{2} y z^{2}+c_{3} z t^{3}+c_{4} t z^{3},
$$



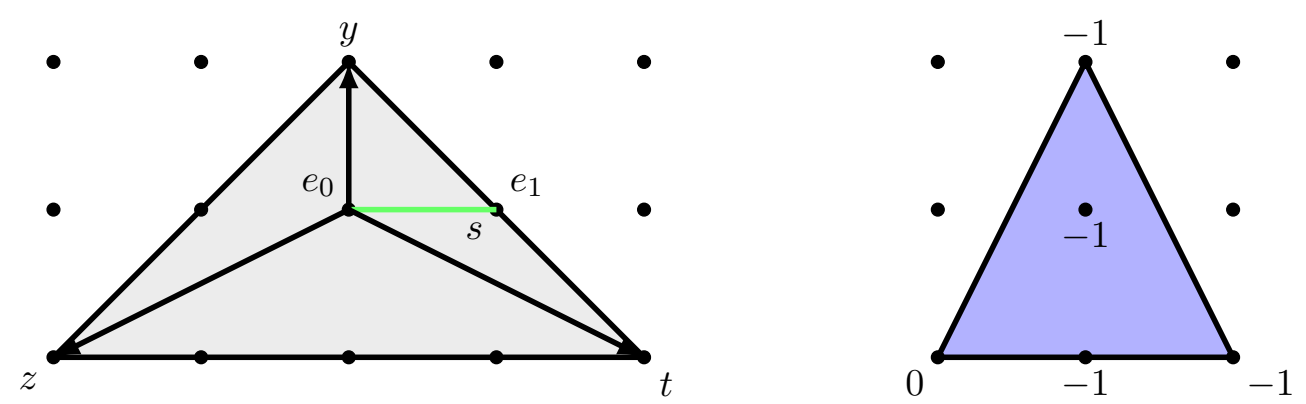

Figure 3. On the lefthand side the only possible $\mathfrak{s u}(2)$-top over polygon 13 of [43] is depicted. The green color indicates the layer at height one, containing the nodes $e_{0}$ and $e_{1}$. On the righthand side we give the dual top, bounded from below by the values $z_{\min }$, shown next to the nodes.

enforcing the $\mathbb{Z}_{2}$ symmetry by setting $c_{i} \equiv 0$ (we call the resulting space $\widetilde{\hat{Y}_{4}^{\prime}}$ ) and then quotienting by this $\mathbb{Z}_{2}$ symmetry. The fact that $\hat{Y}_{4}^{\prime}$ is really the quotient of $\widetilde{\hat{Y}}_{4}^{\prime}$ by (4.24) is automatically implemented by the toric description because the dual polyhedron exclusively contains monomials invariant under (4.24). Importantly, the $\mathbb{Z}_{2}$ acts freely as the fixed point sets $\{t=y=0\}$ and $\{z=y=0\}$ do not lie on $\hat{Y}_{4}^{\prime}$ due to the Stanely-Reisner ideal. Note that the role of this $\mathbb{Z}_{2}$ quotient symmetry was stressed already in [41] albeit in a slightly different context.

This description makes the existence of discrete one-cycles on $\hat{Y}_{4}^{\prime}$ manifest: consider the locus $z=0$ on (4.23). On $\widetilde{\hat{Y}_{4}^{\prime}}$ it is given by $y= \pm 1$, where we have used the scaling of $\mathbb{P}_{1,1,2}$ to set $t=1$ since $t$ and $z$ cannot simultaneously vanish as a consequence of the Stanely-Reisner ideal. A path from $y=-1$ to $y=+1$ on the double cover $\widetilde{\hat{Y}_{4}^{\prime}}$ corresponds to a non-contractible closed loop on $\hat{Y}_{4}^{\prime}$. This loop is torsional as going along it twice is contractible again.

The existence of a torsion one-cycle implies also a torsion six-cycle because in general

$$
\operatorname{Tor}_{p}(Y) \simeq \operatorname{Tor}_{D-p-1}(Y)
$$

with $D$ the real dimension of $Y$. This picture has relied on setting $s=1$ and is thus really valid away from the locus $a_{4}=0$. Therefore all we can conclude is the existence of a 2-torsion element in $H^{1,1}\left(\hat{Y}_{4}, \mathbb{Z}\right) /\langle S\rangle_{\mathbb{Z}}$.

\subsection{An $(\mathrm{SU}(2) \times \mathrm{SU}(2)) / \mathbb{Z}_{2}$-fibration}

The analysis so far has treated all coefficients $a_{i}$ appearing in (4.1) as maximally generic. We now further restrict the coefficients $a_{i}$ defining the $\mathbb{Z}_{2}$-torsional fibration in its singular form (4.1) or its resolution (4.9) such as to create additional non-abelian singularities in the fiber. A special class of such restrictions corresponds to specializations $a_{i} \rightarrow a_{i, j} w^{j}$ with $W:\{w=0\}$ a base divisor and $a_{i, j}$ generic. Since the fibration (4.1) is in global Tate form, the possible enhancements one can obtain via such specialisations can be conveniently determined via Tate's algorithm [58-60] as summarized e.g. in table 2 of [60]. Another advantage of this class of enhancements is that the corresponding fibrations can be treated torically. Indeed, the possible enhancements of type $a_{i} \rightarrow a_{i, j} w^{j}$ with generic $a_{i, j}$ which 
admit a crepant resolution are classified by the tops construction [42, 61], which provides both the possible vanishing patterns $a_{i, j}$ (coinciding with Tate's algorithm) and the toric resolution. For a detailed account of how to read off the vanishing orders from the toric data of a top in the present context we also refer to [17].

From the classification of tops by Bouchard and Skarke [43] for the 16 hypersurface elliptic fibrations, we note that the only tops possible for the fiber (4.7) correspond to singularity type $A_{2 n+1}$ for $n \geq 0, C_{n}$ and $D_{2 n+4}$ for $n \geq 1, B_{3}$ and $E_{7}$. This is indeed in agreement with an analysis via Tate's algorithm as a consequence of $a_{3} \equiv 0$ and $a_{6} \equiv 0$. The associated gauge algebras have the property that their universal cover groups have a center with a $\mathbb{Z}_{2}$-subgroup. Indeed, as we will exemplify below, in all models of this type the Mordell-Weil torsion $\mathbb{Z}_{2}$ will be identified with this $\mathbb{Z}_{2}$-subgroup of the center.

To verify this pattern explicitly we begin with an $A_{1}$ top, corresponding to an affine $\mathfrak{s u}(2)$ type fiber over a divisor $W: w=0$ on $\mathcal{B}$. There is, in fact, only one possible $A_{1}$ top over this polygon, see figure 3 . The singular version of the associated fibration is obtained by replacing in (4.1) $a_{4}$ by $a_{4,1} w$. The discriminant of this fibration,

$$
\Delta \sim w^{2} a_{4,1}^{2}\left(\left(a_{1}^{2}-4 a_{2}\right)^{2}-64 w a_{4,1}\right),
$$

reflects the gauge algebra $\mathfrak{s u}(2) \oplus \mathfrak{s u}(2)$.

The toric resolution of this fibration is described by the hypersurface equation

$$
\hat{P}=s y^{2}+a_{1} s t y z-e_{1} s^{2} t^{4}-a_{2} s t^{2} z^{2}-a_{4,1} e_{0} z^{4},
$$

corresponding to the reflexive pair in figure 3 (again after scaling $x$ to one, since $X$ does not intersect the hypersurface). For definiteness we choose a triangulation with StanleyReisner ideal

$$
\left\{s z, t z, t y, e_{0} s, e_{1} z\right\} .
$$

The extra $\mathfrak{s u}(2)$-fiber is found over $W:\{w=0\}$ with $\pi^{*} w=e_{0} e_{1}$. Indeed, over $W$ the two fiber components $\mathbb{P}_{0}^{1}$ and $\mathbb{P}_{1}^{1}$ are given by the intersection of the ambient divisors $E_{0}:\left\{e_{0}=0\right\}$ and $E_{1}:\left\{e_{1}=0\right\}$ with the hypersurface equation and two generic divisors in the base,

$$
\mathbb{P}_{i}^{1}=\left.E_{i} \cap \hat{P}\right|_{e_{i}=0} \cap D_{a} \cap D_{b}, \quad i=0,1 .
$$

They intersect as the affine $\mathfrak{s u}(2)$ Dynkin diagram.

The discriminant also suggests three codimension-two enhancement loci, at $W \cap\left\{a_{4,1}=\right.$ $0\}, W \cap\left\{a_{1}^{2}=4 a_{2}\right\}$ and $\left\{a_{4,1}=0\right\} \cap\left\{a_{1}^{2}=4 a_{2}\right\}$. Splitting of fiber components only occurs over the first one, ${ }^{7}$ where $\mathbb{P}_{1}^{1}$ factors into the two components

$$
\begin{aligned}
& \mathbb{P}_{1 s}^{1}=E_{1} \cap\{s=0\} \cap\left\{a_{4,1}=0\right\} \cap D_{a} \cap D_{b}, \\
& \mathbb{P}_{1 A}^{1}=E_{1} \cap\left\{\left(y+\frac{1}{2} a_{1} t \pm t \sqrt{\frac{a_{1}^{2}}{4}-a_{2}}\right)\left(y+\frac{1}{2} a_{1} t \mp t \sqrt{\frac{a_{1}^{2}}{4}-a_{2}}\right)=0\right\} \\
& \cap\left\{a_{4,1}=0\right\} \cap D_{a} \cap D_{b} .
\end{aligned}
$$

\footnotetext{
${ }^{7}$ The other two loci are completely analogous to the curve $\left\{a_{4}=0\right\} \cap\left\{a_{1}^{2}=4 a_{2}\right\}$ analysed in the previous section, where no splitting of the fiber was found despite an enhancement of the vanishing order of the discriminant.
} 

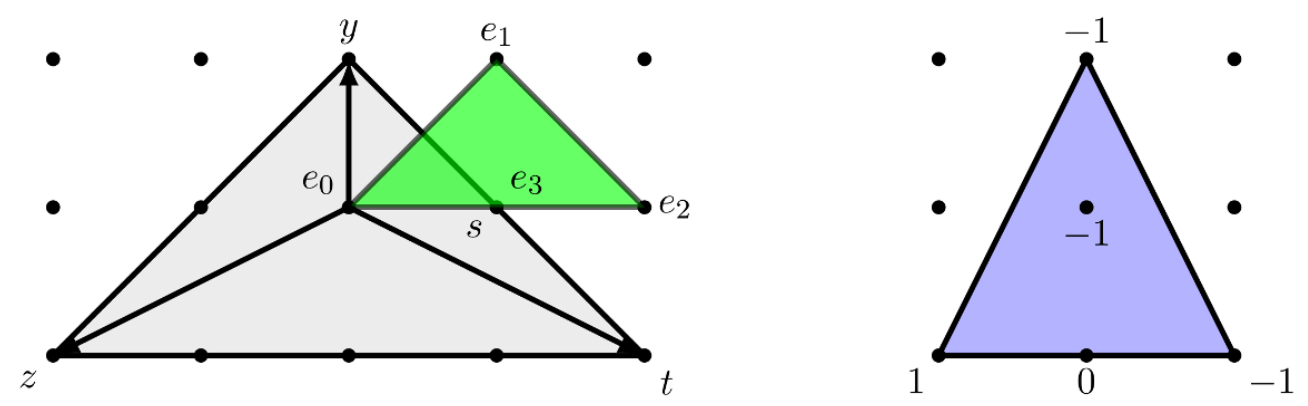

Figure 4. The lefthand side shows an $\mathfrak{s u}(4)$-top over polygon 13 of [43]. The green layer contains the points at height one. On the righthand side we depict the dual top, bounded from below by the values $z_{\min }$, shown next to the nodes.

Note that the two factors in brackets appearing in $\mathbb{P}_{1 A}^{1}$ get exchanged when the sign of the square root changes across a branch cut on $\mathcal{B}$ so that $\mathbb{P}_{1 A}^{1}$ really describes a single $\mathbb{P}^{1}$. The weight

$$
\mathbb{P}_{1 A}^{1} \cdot\left(E_{1}, S\right)=(-1,1)
$$

is in the weight system of the $(\mathbf{2}, \mathbf{2})$ of $\mathfrak{s u}(2) \oplus \mathfrak{s u}(2)$. This implies massless matter in the $(\mathbf{2}, \mathbf{2})$ representation over $W \cap\left\{a_{4,1}=0\right\}$. Again, no fundamental matter $(\mathbf{1}, \mathbf{2})$ or $(\mathbf{2}, \mathbf{1})$ is found.

Our derivation of the extra coweight induced by the torsional section $T:\{t=0\}$ is only mildly modified by the extra $\mathfrak{s u}(2)$ singularity compared to the previous section. The Shioda map $\Sigma_{2}$ of $T$ takes the form

$$
\Sigma_{2}=T-Z-\overline{\mathcal{K}}+\frac{1}{2}\left(S+E_{1}\right),
$$

which is trivial on the hypersurface since the divisor class

$$
X=2 Z-S-2 T+2 \overline{\mathcal{K}}-E_{1}
$$

does not intersect (4.29) due to the Stanley-Reisner ideal. The extra coweight is associated with the class

$$
\Xi_{2} \equiv T-Z-\overline{\mathcal{K}}=-\frac{1}{2}\left(S+E_{1}\right)
$$

which is torsion in $H^{1,1}\left(\hat{Y}_{4}, \mathbb{Z}\right) /\left\langle S, E_{1}\right\rangle_{\mathbb{Z}}$ and manifestly integral on the split curves over $W \cap\left\{a_{4,1}=0\right\}$. This explains why the bifundamental representation is indeed present, whereas fundamental representations of the form $(\mathbf{1}, \mathbf{2})$ or $(\mathbf{2}, \mathbf{1})$, which for group theoretic reasons would have fractional pairing with the coweight $\Xi_{2}$, are not possible.

This refinement of the coweight lattice makes the gauge group non-simply connected and the gauge group is

$$
G=\frac{\mathrm{SU}(2) \times \mathrm{SU}(2)}{\mathbb{Z}_{2}} .
$$

An example of this type was also given in [20]. 


\subsection{An $(\mathrm{SU}(4) \times \mathrm{SU}(2)) / \mathbb{Z}_{2}$-fibration}

In this section we consider the next example in the $A$-series [43], corresponding to an affine $\mathfrak{s u}(4)$-type fiber. This construction yields the unique top of figure 4 associated with the hypersurface equation

$$
\hat{P}=-y^{2} s e_{1}-a_{1} y z s t+s^{2} t^{4} e_{2}^{2} e_{3}+a_{2,1} z^{2} s t^{2} e_{0} e_{2} e_{3}+a_{4,2} z^{4} e_{0}^{2} e_{3} .
$$

The pullback of the projection of the fibration obeys $e_{0} e_{1} e_{2} e_{3}=\pi^{*} w$, defining an affine $\mathfrak{s u ( 4 )}$ fiber over $W:\{w=0\}$ in the base. From one of the 16 triangulations of this top we obtain the Stanley-Reisner ideal

$$
\text { SR-i : } \quad\left\{y t, y e_{0}, y e_{2}, y e_{3}, s z, s e_{0}, s e_{2}, s e_{3}, z e_{2}, z e_{3}, e_{0} e_{2}, t z e_{1}, t e_{0} e_{1}, t e_{1} e_{3}\right\} \text {. }
$$

The three exceptional divisors $e_{1}, e_{2}, e_{3}$ and the part of the original fiber $e_{0}$ are all fibered over $\{w=0\}$ with fiber components

$$
\mathbb{P}_{i}^{1}=\left\{E_{i}\right\} \cap\left\{\left.\hat{P}\right|_{e_{i}=0}=0\right\} \cap D_{a} \cap D_{b} \quad i=0, \ldots, 3 .
$$

The explicit equations are provided in appendix A.1. The irreducible fiber components intersect like the nodes of the affine Dynkin diagram of $\mathfrak{s u}(4)$ type. This is also seen in figure 4 , where the upper layer reproduces this structure by construction. To analyze the localised charged matter we infer from the discriminant of (4.38),

$$
\Delta=16 w^{4} a_{4,2}^{2}\left(\left(4 w a_{2,1}+a_{1}^{2}\right)^{2}-64 w^{2} a_{4,2}\right),
$$

the codimension-two enhancement loci ${ }^{8}$

$$
\left\{w=a_{4,2}=0\right\} \quad \text { and } \quad\left\{w=a_{1}=0\right\} .
$$

The factorization properties of the fiber components (see appendix A.1) identify the split curves in the fiber. At $\left\{w=a_{4,2}=0\right\}$ the component $\mathbb{P}_{1}^{1}$ splits into three components, whose intersection numbers with the exceptional divisors from the $\mathfrak{s u}(4)$ and $\mathfrak{s u}(2)$ singularities are

$$
\begin{array}{ll}
\mathbb{P}_{e_{1}=s=0}^{1} \cdot\left(E_{1}, E_{2}, E_{3}\right)=(0,0,0), & \mathbb{P}_{e_{1}=s=0}^{1} \cdot(S)=(-2), \\
\mathbb{P}_{e_{1}=t=0}^{1} \cdot\left(E_{1}, E_{2}, E_{3}\right)=(-1,1,0), & \mathbb{P}_{e_{1}=t=0}^{1} \cdot(S)=(1), \\
\mathbb{P}_{e_{1}=R 1=0}^{1} \cdot\left(E_{1}, E_{2}, E_{3}\right)=(-1,0,0), & \mathbb{P}_{e_{1}=R 1=0}^{1} \cdot(S)=(1),
\end{array}
$$

respectively. The $(-1,1,0)$ and $(-1,0,0)$ are weights in the fundamental of $\mathfrak{s u}(4)$ and from the right column we find the weights of the fundamental representation of $\mathfrak{s u}(2)$ (which is the same as the anti-fundamental). Indeed the full weight system is reproduced by taking linear combinations of fibral curves. Hence the charged matter at this locus transforms in representation $(\mathbf{4}, \mathbf{2})$ of $\mathfrak{s u}(4) \oplus \mathfrak{s u}(2)$.

\footnotetext{
${ }^{8}$ All other enhancement loci as read off from the discriminant do not correspond to an extra fiber splitting.
} 
Over $\left\{w=a_{1}=0\right\}$ the relevant intersections are

$$
\begin{aligned}
& \mathbb{P}_{e_{1}=e_{3}=0}^{1} \cdot\left(E_{1}, E_{2}, E_{3}\right)=(0,1,-2), \quad \mathbb{P}_{e_{1}=e_{3}=0}^{1} \cdot(S)=(0), \\
& \mathbb{P}_{e_{1}=R 2_{1}=0}^{1} \cdot\left(E_{1}, E_{2}, E_{3}\right)=(-1,0,1), \quad \mathbb{P}_{e_{1}=R 2_{1}=0}^{1} \cdot(S)=(0), \\
& \mathbb{P}_{e_{1}=R 2_{2}=0}^{1} \cdot\left(E_{1}, E_{2}, E_{3}\right)=(-1,0,1), \quad \mathbb{P}_{e_{1}=R 2_{2}=0}^{1} \cdot(S)=(0),
\end{aligned}
$$

where $(-1,0,1)$ is one of the weights in the $\mathbf{6}$-representation of $\mathfrak{s u}(4)$. States originating from these curves are uncharged under $\mathfrak{s u}(2)$. This is as expected since this locus is away from the $\mathfrak{s u}(2)$ divisor $\left\{a_{4,2}=0\right\}$. The following table summarizes the matter spectrum:

\begin{tabular}{|cc|}
\hline \multicolumn{2}{|c|}{$\begin{array}{c}\text { Top over polygon 13: } \\
\mathfrak{s u}(4) \times \mathfrak{s u}(2)\end{array}$} \\
\hline Locus & Charged matter \\
$w \cap a_{4,2}$ & $(\mathbf{4}, \mathbf{2})$ \\
$w \cap a_{1}$ & $(\mathbf{6}, \mathbf{1})$ \\
\hline
\end{tabular}

Again we stress the absence of fundamental representations. The Shioda-type Ansatz for the toric divisor class $T$ yields

$$
\Sigma_{2}=T-Z-\overline{\mathcal{K}}+\frac{1}{2}\left(S+E_{1}+2 E_{2}+E_{3}\right),
$$

which for the same reasons as before turns out to be trivial in $H^{1,1}\left(Y_{4}, \mathbb{R}\right)$. The coweight element

$$
\Xi_{2}=T-Z-\overline{\mathcal{K}}=-\frac{1}{2}\left(S+E_{1}+2 E_{2}+E_{3}\right),
$$

which is 2-torsion in $H^{1,1}\left(\hat{Y}_{4}, \mathbb{Z}\right) /\left\langle S, E_{1}, E_{2}, E_{3}\right\rangle_{\mathbb{Z}}$, forces the weight lattice to be coarser in order to preserve the integer pairing of coweights and weights. Indeed, the intersection of $\Xi_{2}$ with all split curves corresponding to weights of the matter representations is integer, and representations such as $(\mathbf{4}, 1)$ or $(1,2)$ which would violate this integral pairing are absent. This identifies the global gauge group as

$$
G=\frac{\mathrm{SU}(4) \times \mathrm{SU}(2)}{\mathbb{Z}_{2}}
$$

\subsection{A $(\operatorname{Spin}(7) \times \mathrm{SU}(2)) / \mathbb{Z}_{2}$-fibration}

Keeping the same ambient fiber space as in previous section, we now consider a top corresponding to the non-simply laced Lie algebra $B_{3}$. The top is constructed uniquely from the classification [43] and the corresponding hypersurface equation is

$$
\hat{P}=e_{1}^{2} s^{2} t^{4}+e_{3} s y^{2}+a_{1} e_{0} e_{1} e_{2} e_{3} s t y z+a_{2} e_{0} e_{1} s t^{2} z^{2}+a_{4} e_{0}^{2} z^{4} .
$$

Having a node at $z=2$ in the top defines the divisor $W=\{w=0\}$ in the base with $\pi^{*} w=e_{0} e_{1} e_{2}^{2} e_{3}$ and gives multiplicity 2 to the corresponding curve $\mathbb{P}_{2}^{1}$ in the fiber over $W$. The occurrence of the multiplicity of the node in the projection to the base is crucial to 

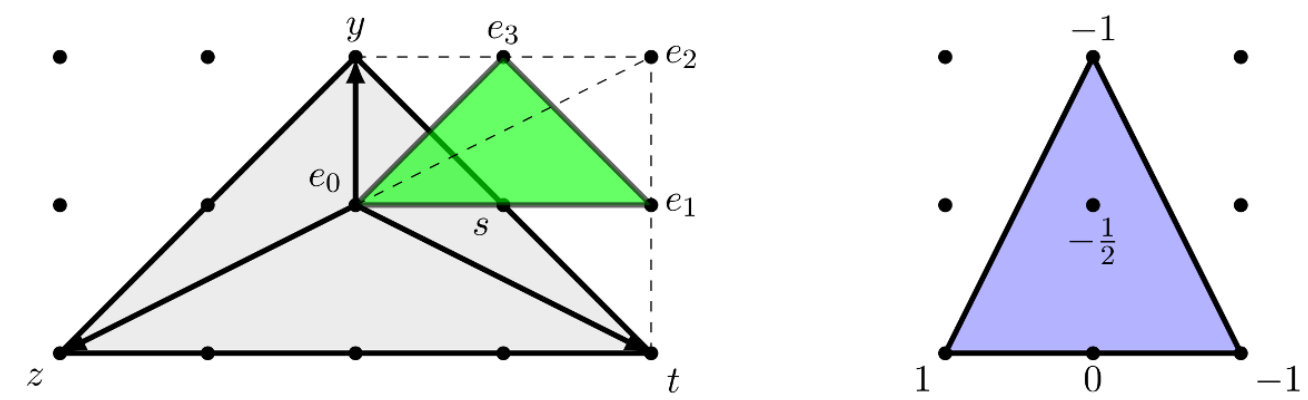

Figure 5. The lefthand side shows the unique $B_{3}$-top over polygon 13 of [43]. The green layer contains the points at height one and the node labelled $e_{2}$ is at height two. On the right side we depict the dual top, bounded from below by the values $z_{\min }$, shown next to the nodes.

make $W$ scale under the scaling relations coming from the $z \geq 1$ layers of the top. The affine $B_{3}$ Dynkin diagram is read off along the edges at $z \geq 1$ of the top in figure 5 . The non-simply laced structure of this algebra is reflected in the fact that the intersection of the ambient divisor $E_{3}$ with the hypersurface

$$
\left.E_{3} \cap \hat{P}\right|_{e_{3}=0} \cap D_{a} \cap D_{b}, \quad D_{a, b} \subset \mathcal{B}
$$

gives rise to two curves. These are described by the factorization

$$
\begin{aligned}
& \left\{e_{3}=0\right\} \cap\left\{s^{2}+a_{2} e_{0} s+a_{4} e_{0}^{2}=0\right\} \Leftrightarrow \\
& \left\{e_{3}=0\right\} \cap\left\{\left(s+\frac{1}{2} a_{2} e_{0} \pm e_{0} \sqrt{\frac{a_{2}^{2}}{4}-a_{4}}\right)\left(s+\frac{1}{2} a_{2} e_{0} \mp e_{0} \sqrt{\frac{a_{2}^{2}}{4}-a_{4}}\right)=0\right\} .
\end{aligned}
$$

The two factors on the righthand side give rise to the curves $\mathbb{P}_{3 \pm}^{1}$ and they get exchanged when the signs of the square roots shift upon travelling along $W$ in the base. As a check, the negative of the Cartan matrix $C_{i j}$ of $B_{3}$ is reproduced as the intersection numbers

$$
E_{i} \cdot\left(\mathbb{P}_{0}^{1}, \mathbb{P}_{1}^{1}, \mathbb{P}_{2}^{1}, \mathbb{P}_{3 \pm}^{1}\right)_{j}=-C_{i j}
$$

By analyzing the codimension-two loci, we find only one curve in $\mathcal{B}$ over which the fiber degenerates further. This happens over $W \cap\left\{a_{4}=0\right\}$ and by calculating the charges of the split fiber components weights in the weight system of the $(\mathbf{8}, \mathbf{2})$ of $\mathfrak{s o}(7) \oplus \mathfrak{s u}(2)$ are found, where $\mathbf{8}$ is the spinor representation.

Using that the toric divisor $X$ does not restrict to the hypersurface the Shioda map of the torsional section gives a class

$$
\Xi_{2} \equiv T-Z-\overline{\mathcal{K}}=-\frac{1}{2}\left(S+2 E_{1}+2 E_{2}+E_{3}\right)
$$

with integer intersection with all fiber components over the matter curve.

Consistently with the appearance of the representation $(\mathbf{8}, \mathbf{2})$ of $\mathfrak{s o}(7) \oplus \mathfrak{s u}(2)$ the gauge group is

$$
G=\frac{\operatorname{Spin}(7) \times \mathrm{SU}(2)}{\mathbb{Z}_{2}}
$$


where the $\mathbb{Z}_{2}$ is the common center of $\operatorname{Spin}(7)$ and $\mathrm{SU}(2)$ and $\pi_{1}(G)=\mathbb{Z}_{2}$. Even though not realized in this geometry, all representations $\left(\mathbf{8}, \mathbf{R}_{\mathbf{e}}\right)$ for $\mathbf{R}_{e}$ an even-dimensional representation of $\mathrm{SU}(2)$ would also be allowed, and also the representations $\left(\mathbf{7}, \mathbf{R}_{o}\right)$ for $\mathbf{R}_{o}$ an odd-dimensional representation of $\mathrm{SU}(2)$.

\subsection{Generalisation to $\operatorname{Sp}(n) / \mathbb{Z}_{2}, \mathrm{SU}(2 n) / \mathbb{Z}_{2}, \operatorname{Spin}(4 n) / \mathbb{Z}_{2}$, Type IIB limit and restricted monodromies}

The toric enhancements described in the previous sections involved the specialization $a_{4} \rightarrow$ $a_{4, n} w^{n}$ for $W:\{w=0\}$ some divisor different from the $A_{1}$-locus $\left\{a_{4}=0\right\}$. Clearly one can also identify $w$ with $a_{4}$, thereby producing a single gauge group factor. According to the general discussion, this single group factor will be strongly constrained by the requirement that the universal cover gauge group $G_{0}$ contain a $\mathbb{Z}_{2}$-subgroup in its center.

Indeed, if $\overline{\mathcal{K}}_{\mathcal{B}}^{4 / n}$ exists as a line bundle with non-trivial sections, we can simply factorise

$$
a_{4}=\left(\tilde{a}_{4}\right)^{n}
$$

with $\tilde{a}_{4} \in H^{0}\left(\mathcal{B}, \overline{\mathcal{K}}_{\mathcal{B}}^{4 / n}\right)$. Since the analysis of the singular geometry and its resolution has been exemplified in detail in the previous sections, we content ourselves with determining the resulting gauge groups by application of Tate's algorithm [58,60] without explicitly constructing the resolution. For generic $a_{2}$, Tate's algorithm in the form of table 2 of [60] indicates that the fiber over $\tilde{a}_{4}=0$ is of Kodaira type $I_{2 n}^{n s}$, with the superscript denoting the non-split type. The associated gauge algebra is the rank $n$ Lie algebra $\mathfrak{s p}(n)$ (with the convention that $\mathfrak{s p}(1) \simeq \mathfrak{s u}(2))$. This identifies the gauge group as

$$
G=\frac{\operatorname{Sp}(n)}{\mathbb{Z}_{2}} .
$$

As described in subsection 4.1.1, if $n=1$ the global structure of $G$ makes extra massless representations along the curve $\left\{\tilde{a}_{4}=0\right\} \cap\left\{\frac{1}{4} a_{1}^{2}+a_{2}=0\right\}$ impossible; this is no longer true for $n \geq 2$. Indeed, in this case Tate's algorithm predicts, as described in detail in [59], for the fiber type over this curve Kodaira type $I_{2 n-4}^{* s}$ (with the superscript standing for split type), corresponding to gauge algebra $\mathfrak{s o}(4 n)$. From the branching rule of the adjoint of $\mathrm{SO}(4 n)$ along $\mathrm{SO}(4 n) \rightarrow \mathrm{SU}(2 n) \times \mathrm{U}(1) \rightarrow \mathrm{Sp}(n) \times \mathrm{U}(1)$ one deduces matter in the 2-index antisymmetric representation of $\operatorname{Sp}(n)$ of dimension $2 n^{2}-n-1$ along $\left\{\tilde{a}_{4}=0\right\} \cap\left\{\frac{1}{4} a_{1}^{2}+a_{2}=\right.$ $0\}$ (see in particular table 9 of [59]). This is compatible with the gauge group $G=\frac{\operatorname{Sp}(n)}{\mathbb{Z}_{2}}$.

Next, one can engineer a gauge algebra $\mathfrak{s u}(2 n)$ by factoring $a_{4}=\left(\tilde{a}_{4}\right)^{n}$ and in addition restricting $a_{2}=a_{2,1} \tilde{a}_{4}$ for suitable $a_{2,1} \in H^{0}\left(\mathcal{B}, \overline{\mathcal{K}}_{\mathcal{B}}^{2-4 / n}\right)$ (if existent). In this case the gauge group is

$$
G=\frac{\mathrm{SU}(2 n)}{\mathbb{Z}_{2}}, \quad n \geq 2 .
$$

Note that the Mordell-Weil torsion group $\mathbb{Z}_{2}$ appears here as a proper subgroup of the center $\mathbb{Z}_{2 n}$ of the universal cover $G_{0}=\mathrm{SU}(2 n)$. The same argument as above predicts massless matter in the antisymmetric representation of $\mathrm{SU}(2 n)$ localised on the curve $\left\{\tilde{a}_{4}=0\right\} \cap$ $\left\{a_{1}=0\right\}$. The appearance of this matter distinguishes $G=\mathrm{SU}(2 n) / \mathbb{Z}_{2}$ as realized here from 
$\mathrm{SU}(2 n) / \mathbb{Z}_{2 n}$. The possibility that the Mordell-Weil torsion appears as a proper subgroup of the center of the universal cover $G_{0}$ had previously been noted in eight-dimensional F-theory compactifications on K3 in [32, 62].

The only remaining chain of enhancements of this type which is possible according to Tate's algorithm leads to gauge algebra $\mathfrak{s o}(4 n)$ with $n \geq 4$ and corresponds to $a_{4}=\left(\tilde{a}_{4}\right)^{n}$, $a_{2}=a_{2,1} \tilde{a}_{4}$ and $a_{1}=a_{1,1} \tilde{a}_{4}$. The restriction to $n \geq 4$ comes about as a necessary condition for a section $a_{1} \in H^{0}\left(\mathcal{B}, \overline{\mathcal{K}}_{\mathcal{B}}^{1-4 / n}\right)$ to exist. According to the analysis in [59] we expect matter in the vector representation along the curve $\left\{\tilde{a}_{4}=0\right\} \cap\left\{a_{2,1}=0\right\}$. Note that the universal cover group $G_{0}=\operatorname{Spin}(4 n)$ has center $\mathbb{Z}_{2} \times \mathbb{Z}_{2}$. The appearance of the vector representation (but not the spinor) is in perfect agreement with the gauge group being

$$
G=\frac{\operatorname{Spin}(4 n)}{\mathbb{Z}_{2}}=\operatorname{SO}(4 n), \quad n \geq 4 .
$$

The observed pattern has a natural interpretation in the weak coupling Type IIB orientifold limit. This Sen limit [63] is realized as the limit $\epsilon \rightarrow 0$ after rescaling $a_{3} \rightarrow \epsilon a_{3}, a_{4} \rightarrow \epsilon a_{4}$, $a_{6} \rightarrow \epsilon^{2} a_{6}$ [64]. The discriminant locus can be brought into the form

$$
\Delta \simeq \epsilon^{2} h^{2}\left(\eta^{2}-h \chi\right)+\mathcal{O}\left(\epsilon^{3}\right),
$$

and the Type IIB Calabi-Yau

$$
X_{\text {IIB }}: \xi^{2}=h
$$

is a double cover of the F-theory base $\mathcal{B}$ branched over the orientifold plane localised at $h=0$. The orientifold action on $X_{\mathrm{IIB}}$ acts as $\xi \rightarrow-\xi$. The locus $\eta^{2}-h \chi=0$ on $\mathcal{B}$ and its uplift to the Calabi-Yau double cover $X_{\text {IIB }}$ represents the D7-brane locus. In the configuration at hand, due to the restriction $a_{3} \equiv 0$ and $a_{6} \equiv 0$, one finds

$$
h=-\frac{1}{12}\left(a_{1}^{2}+4 a_{2}\right), \quad \chi=0, \quad \eta=a_{4}=\left(\tilde{a}_{4}\right)^{n} .
$$

For generic $a_{2}$ the D7-brane system is given by a stack of D7-branes on the uplift of the divisor $\left\{a_{4}=0\right\}$ to the double cover $X_{\mathrm{IIB}}$; since this locus is invariant under the orientifold projection, the D7-brane stack supports gauge algebra $\mathfrak{s p}(n)$. The antisymmetric matter appears at the intersection with the O7-plane at $h=0$. If $a_{2}=a_{2,1} \tilde{a}_{4}$, then the analysis of [8] shows that the D7-branes wrap a divisor on the Calabi-Yau double cover which is not mapped to itself under the orientifold action. Its corresponding non-abelian gauge algebra is therefore indeed $\mathfrak{s u}(n)$ with antisymmetric matter at the intersection of the D7-brane stack with its image on top of the O7-plane. For completeness, note that the further specialization $a_{1}=a_{1,1} \tilde{a}_{4}$, corresponding to the $\operatorname{Spin}(4 n) / \mathbb{Z}_{2}$ series in F-theory, has an ill-defined weak-coupling limit with two O7-planes intersecting over a curve of conifold singularities.

Apart from reproducing the F-theory predictions, this weak coupling analysis exemplifies how the global structure of the gauge group in the Type IIB limit can be understood from the specific D7-brane configuration and the absence (or presence) of certain matter representations. In the situation under consideration, what changes the gauge group from 
$\mathrm{Sp}(n)$ or $\mathrm{SU}(2 n)$ to $\mathrm{Sp}(n) / \mathbb{Z}_{2}$ and $\mathrm{SU}(2 n) / \mathbb{Z}_{2}$ is that in the discriminant (4.59) no extra single D7-brane arises in addition to the non-abelian brane stack at $\left\{\tilde{a}_{4}=0\right\}$; if present the intersection curve of such a brane with the D7-brane stack would lead to matter in the fundamental representation of $\mathrm{Sp}(n)$ or $\mathrm{SU}(2 n)$ and thus change the global structure of the gauge group.

Finally, let us point out that the elliptic fibration (4.9) with $s \equiv 1$, i.e. the singular model corresponding to the blow-down of the $A_{1}$-fiber at $\left\{a_{4}=0\right\}$, was considered in [41] from a related, but slightly different perspective: in this work it was shown that this class of elliptic fibrations does not exhaust the full $\mathrm{SL}(2, \mathbb{Z})$ monodromy group, but only the subgroup $\Gamma_{0}(2) \subset \mathrm{SL}(2, \mathbb{Z}) .{ }^{9}$ In fact, restricted $\Gamma_{0}(k)$-monodromy is a consequence of the existence of an order $k$ point on the elliptic fiber [41], which, in the language of our analysis, is equivalent to Mordell-Weil $k$-torsion. There are a number of geometric consequences of this [44]. For example, the modular curve $\mathfrak{h} / \Gamma_{0}(2)$ has two "cusp" points at which $j=\infty$, corresponding to the two irreducible factors $a_{4}$ and $\left(4 a_{4}-\left(a_{2}+\frac{1}{4} a_{1}^{2}\right)^{2}\right)$ of the discriminant (4.2). As we have seen in examples, it is the factor $a_{4}$ which vanishes when the corresponding gauge group factor is related to $\mathbb{Z}_{2}$ torsion. By contrast, one can in principle also engineer additional gauge group factors by factorising $\left(4 a_{4}-\left(a_{2}+\frac{1}{4} a_{1}^{2}\right)^{2}\right)$ without factorising $a_{4}$ as such. Such non-toric enhancements would lead to what we called the 'spectator' gauge group $G^{\prime}$ in section 3.3 and which is unconstrained by the $\mathbb{Z}_{2}$ torsion. Indeed, while all gauge algebras that can be engineered torically are easily checked to lead to Kodaira monodromies contained in $\Gamma_{0}(2)$, this set does not exhaust the list of $\Gamma_{0}(2)$ compatible singularities (e.g. it misses $A_{2 k}$ - see appendix B of [41]). Such algebras would have to come from a non-toric enhancement involving the second factor of the discriminant. We will see an example of an abelian spectator group $G^{\prime}=\mathrm{U}(1)$ in the next section.

\section{$5 \quad$ Mordell-Weil group $\mathbb{Z} \oplus \mathbb{Z}_{2}$}

\subsection{An $(\mathrm{SU}(2) \times \mathrm{SU}(2)) / \mathbb{Z}_{2} \times \mathrm{U}(1)$ fibration}

The generic elliptic fibration with $\mathbb{Z}_{2}$-torsional Mordell-Weil group admits an interesting specialization such as to enhance the Mordell-Weil group to $\mathbb{Z}_{2} \oplus \mathbb{Z}$. As it turns out the generator of the free part of the Mordell-Weil group can be described again very conveniently as a toric section.

In fact, the specialization we have in mind gives rise to the second of the three elliptic fibrations realized as hypersurfaces in a toric ambient space with Mordell-Weil torsion [15]. The fiber is defined by the reflexive pair in figure 6 , which corresponds to polygon 15 and its dual in the classification of [43]. The associated elliptic curve is the vanishing locus of a biquadric in a blow-up of $\mathbb{P}^{1} \times \mathbb{P}^{1}$. The hypersurface equation defined via the dual polygon is

$$
\hat{P}=c d^{2} v^{2} w^{2}+c^{2} d u^{2} v^{2}+\gamma_{1} c d u v w z+\gamma_{2} d w^{2} z^{2}+\delta_{2} c u^{2} z^{2}
$$

\footnotetext{
${ }^{9}$ Recall that $\Gamma_{0}(k)$ is defined as the subgroup of $\operatorname{SL}(2, \mathbb{Z})$-matrices $\left(\begin{array}{ll}a & b \\ c & d\end{array}\right)$ with $c \equiv 0 \bmod k$.
} 

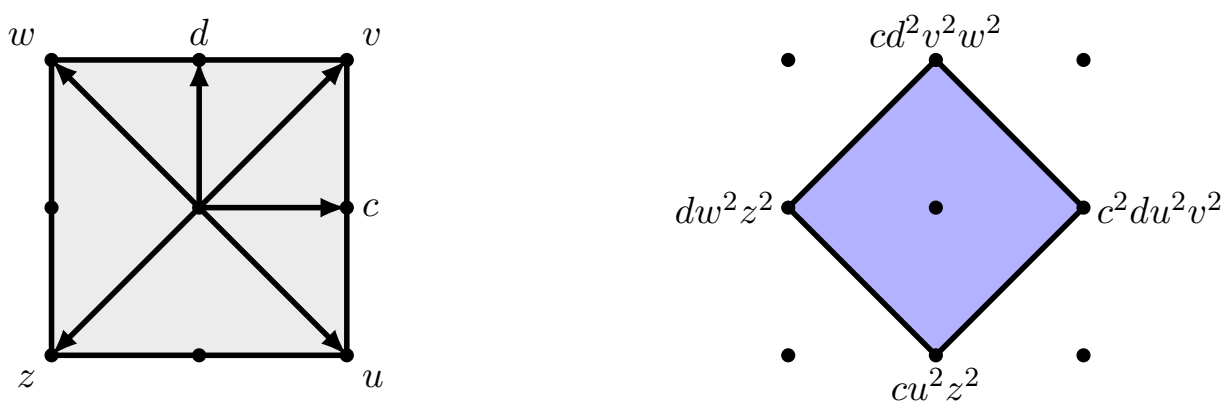

Figure 6. Polygon 15 of [43] together with its dual polygon.

where we have set the coefficients of the first two terms to one since they are sections of the trivial bundle over the base. ${ }^{10}$ The coefficients $\gamma_{i}$ and $\delta_{i}$ are sections of $\overline{\mathcal{K}}^{i}$. A choice for the scaling relations of the fiber coordinates is

\begin{tabular}{|c|c|c|c|c|c|c|}
\hline$u$ & $v$ & $w$ & $z$ & $c$ & $d$ & $\sum$ \\
\hline 1 & 0 & 1 & 0 & 0 & 0 & 2 \\
\hline 0 & 1 & 0 & 1 & 0 & 0 & 2 \\
\hline 0 & 0 & 0 & 1 & 1 & 1 & 3 \\
\hline 0 & 0 & 1 & 1 & 2 & 0 & 4 \\
\hline
\end{tabular}

which is consistent with the degree of homogeneity of (5.1). The Stanley-Reisner ideal of the toric ambient space of the fiber takes the form $\{u v, u w, u d, v z, z c, z d, w c, c d, v w\}$.

The biquadric (5.1) can be brought into Weierstrass form, where it can be compared with the Weierstrass model associated with the fibration (4.1) analysed in the previous section. This identifies

$$
a_{1}=\gamma_{1}, \quad a_{2}=-\left(\gamma_{2}+\delta_{2}\right), \quad a_{4}=\gamma_{2} \delta_{2},
$$

where $a_{i}$ are the coefficients of the generic $\mathbb{Z}_{2}$-torsion fibration (4.1). As we will show, the result of this specialization of $a_{2}$ and $a_{4}$ is the enhancement of the Mordell-Weil group from $\mathbb{Z}_{2}$ to $\mathbb{Z}_{2} \oplus \mathbb{Z}$ (as computed previously in [15]) and the appearance of an extra $\mathfrak{s u}(2)$ factor.

To analyse the non-abelian sector, we first note that the discriminant of equation (5.1) takes the form

$$
\Delta \sim \gamma_{2}^{2} \delta_{2}^{2}\left[\gamma_{1}^{4}-8 \gamma_{1}^{2}\left(\gamma_{2}+\delta_{2}\right)+16\left(\gamma_{2}-\delta_{2}\right)^{2}\right]
$$

Together with the Weierstrass functions $f$ and $g$ of the associated Weierstrass model this suggests an $A_{1}$ singularity at $\left\{\gamma_{2}=0\right\}$ and $\left\{\delta_{2}=0\right\}$ respectively. Indeed, the hypersurface equation factorises over these loci as

$$
\begin{array}{ll}
\left\{\gamma_{2}=0\right\}: & c\left(c d u^{2} v^{2}+d^{2} v^{2} w^{2}+\gamma_{1} d u v w z+\delta_{2} u^{2} z^{2}\right), \\
\left\{\delta_{2}=0\right\}: & d\left(c d v^{2} w^{2}+c^{2} u^{2} v^{2}+\gamma_{1} c u v w z+\gamma_{2} w^{2} z^{2}\right),
\end{array}
$$

and we identify the irreducible components $\mathbb{P}_{c}^{1}$ and $\mathbb{P}_{d}^{1}$ as the restriction to the fiber of the resolution divisors $C:\{c=0\}$ and $D:\{d=0\}$ of these singularities.

\footnotetext{
${ }^{10}$ If we had chosen a fibration such that these two coefficients are sections of non-trivial bundles, $z=0$ would not be a holomorphic section but a birational one.
} 
On general grounds [15,48], the intersection of the toric divisors $U:\{u=0\}, V$ : $\{v=0\}, W:\{w=0\}, Z:\{z=0\}$ with the hypersurface give rise to sections of the fibration, not all of which are independent. Since $Z:\{z=0\}$ is a holomorphic section we choose it as the zero-section. Then, the Mordell-Weil group is generated by differences of sections $U-Z, V-Z, W-Z$, which are not all independent. Let us first consider the Shioda map for the section $U:\{u=0\}$. Requiring, as usual, one leg in the fiber as well as orthogonality with the exceptional divisors gives

$$
W_{U}=2(U-Z-\overline{\mathcal{K}})+C
$$

which is unique up to an overall normalization, here chosen such as to arrive at integer charges below. We take this non-trivial element $W_{U}$ as the generator of the free part of the Mordell-Weil group, and physically identify it with the generator of the associated, suitably normalized $\mathrm{U}(1)$ part of the gauge group.

On the other hand, the intersection of the section $V:\{v=0\}$ with the elliptic curve describes a 2-torsion point, as noted already in [15]. The Shioda map for $V:\{v=0\}$ yields the element

$$
\Sigma_{2}=V-Z-\overline{\mathcal{K}}+\frac{1}{2}(C+D) .
$$

However, $V$ is not an independent toric divisor class, but may be expressed as

$$
V=Z+\overline{\mathcal{K}}-\frac{1}{2}(C+D),
$$

which makes $\Sigma_{2}$ a trivial class. Since the model we consider here is a restriction of the model with just a $\mathbb{Z}_{2}$ section we have the analogous situation that $\Sigma_{2}$ is given by a divisor in the ambient space which restricts to a trivial class on the hypersurface. The integer class

$$
\Xi_{2} \equiv V-Z-\overline{\mathcal{K}}=\frac{1}{2}(C+D)
$$

is 2-torsion in $H^{1,1}\left(\hat{Y}_{4}, \mathbb{Z}\right)$ modulo resolution classes and to be identified with a coweight element momentarily.

Having established the gauge algebra $\mathfrak{s u}(2) \oplus \mathfrak{s u}(2) \oplus \mathfrak{u}(1)$ we turn to the matter representations in codimension 2. From the discriminant (5.4) the three potential enhancement loci which could host matter charged under the non-abelian gauge groups are identified as

$$
\left\{\gamma_{2}=\delta_{2}=0\right\}, \quad\left\{\gamma_{1}=\gamma_{2}=0\right\}, \quad\left\{\gamma_{1}=\delta_{2}=0\right\} .
$$

At the loci $\left\{\gamma_{1}=\gamma_{2}=0\right\}$ and $\left\{\gamma_{1}=\delta_{2}=0\right\}$, which would naively give rise to fundamental matter, the equation does not factorize further, and hence no extra matter is found there. But at the locus $\left\{\gamma_{2}=\delta_{2}=0\right\}$ the equation factorizes as

$$
c d v \underbrace{\left(c u^{2} v+d v w^{2}+\gamma_{1} u w z\right)}_{R},
$$

where the curves $\mathbb{P}_{c}^{1}, \mathbb{P}_{d}^{1}, \mathbb{P}_{v}^{1}$ and the last component $\mathbb{P}_{R}^{1}$ intersect as the affine $A_{3}$ Dynkin diagram. We calculate the charges of the split component $\mathbb{P}_{v=0}^{1}$ as

$$
\mathbb{P}_{v=0}^{1} \cdot(C, D)=(1,1),
$$


giving the highest weights of the bifundamental $(\mathbf{2}, \mathbf{2})$. By acting on this with the respective roots the entire $(\mathbf{2}, \mathbf{2})$ is reproduced. With the normalization (5.6) the $\mathrm{U}(1)$ charge of this state is

$$
W_{U} \cdot \mathbb{P}_{v=0}^{1}=1 .
$$

Extra massless matter is localized at the singlet curve $\left\{\gamma_{2}=\delta_{2}\right\} \cap\left\{\gamma_{1}=0\right\}$. This is an $I_{2}$ locus over which the hypersurface equation factorizes as

$$
\left(c u^{2}+d w^{2}\right)\left(c d v^{2}+\delta_{2} z^{2}\right)
$$

and we denote the fiber components by $\mathbb{P}_{-}^{1}$ and $\mathbb{P}_{+}^{1}$ respectively. These have zero intersection with the Cartan divisors $C, D$ (and are thus invariant also under the center of the gauge group) and their $\mathrm{U}(1)$-charges are computed as

$$
W_{U} \cdot \mathbb{P}_{ \pm}^{1}= \pm 2
$$

Hence we find a representation $(\mathbf{1}, \mathbf{1})_{ \pm 2}$ with respect to $\mathfrak{s u}(2)_{C} \oplus \mathfrak{s u}(2)_{D} \oplus \mathrm{U}(1)$.

At the intersection points $\gamma_{1}=\gamma_{2}=\delta_{2}=0$ of the two matter curves the fiber type changes to form a non-affine Dynkin diagram of $D_{4}$. This is because the component $\left(c u^{2} v+d v w^{2}+\gamma_{1} u w z\right)_{R}$ in the fiber over the curve $\left\{\gamma_{2}=\delta_{2}=0\right\}$ splits off a factor of $v$ as $\gamma_{1}=0$, corresponding to a factorisation

$$
c d v^{2}\left(c u^{2}+d w^{2}\right)
$$

At those points a Yukawa coupling $(\mathbf{2}, \mathbf{2})_{1}(\mathbf{2}, \mathbf{2})_{1}(\mathbf{1}, \mathbf{1})_{-2}$ is localised.

As is manifest, the divisor $\Xi_{2}$ has integer pairing with all split curves associated with the representations $(\mathbf{2}, \mathbf{2})_{1}$ and $(\mathbf{1}, \mathbf{1})_{ \pm 2}$ and is therefore identified with a coweight. With coefficients in $\frac{1}{2} \mathbb{Z}$ the coweight lattice is made finer by this extra coweight, and only weights in representations integer paired with $\Xi_{2}$ are allowed. Again this is the reason for the absence of for example a fundamental representation at the loci $\left\{\gamma_{1}=\gamma_{2}=0\right\}$ and $\left\{\gamma_{1}=\right.$ $\left.\delta_{2}=0\right\}$. Note that the expression for $\Sigma_{2}$ does not include a term proportional to the $\mathrm{U}(1)$-generator $W_{U}$, but only the generators $C$ and $D$ of the $\mathfrak{s u}(2)_{C} \oplus \mathfrak{s u}(2)_{D}$ Cartan U(1)s. In particular, integrality of the pairing of $\Xi_{2}$ does therefore not constrain the allowed U(1) charges, but only the non-abelian part of the representation. We conclude that the gauge group is

$$
G=\frac{\mathrm{SU}(2)_{C} \times \mathrm{SU}(2)_{D}}{\mathbb{Z}_{2}} \times \mathrm{U}(1)
$$

whose first fundamental group $\pi_{1}(G)=\mathbb{Z} \oplus \mathbb{Z}_{2}$ coincides with the Mordell-Weil group as expected.

\subsection{A chain of fibrations via Higgsing}

The elliptic fibrations described in sections 4.1, 4.2 and 5.1 can be viewed as a successive specialization of a Tate model

$$
P=y^{2}-x^{3}+a_{1} x y z+a_{2} x^{2} z^{2}+a_{3} y z^{3}+a_{4} x z^{4}+a_{6} z^{6},
$$


which for generic $a_{i} \in H^{0}\left(\mathcal{B}, \overline{\mathcal{K}}^{i}\right)$ has trivial Mordell-Weil and gauge group. If $a_{6} \equiv 0$, the fibration corresponds to a $\mathrm{U}(1)$ restricted Tate model [4] with Mordell-Weil group $\mathbb{Z}$, gauge group $G=\mathrm{U}(1)$ and a massless singlet $\mathbf{1}_{ \pm 1}$ localized at the curve $\left\{a_{3}=0\right\} \cap\left\{a_{4}=0\right\}$. The extra section degenerates to a $\mathbb{P}^{1}$ over this matter curve $[4,5,8]$. From this, one reaches the fibration (4.1) with Mordell-Weil group $\mathbb{Z}_{2}$ and $G=\mathrm{SU}(2) / \mathbb{Z}_{2}$ by setting in addition $a_{3} \equiv 0$. This promotes the $\mathrm{U}(1)$ generator of the $\mathrm{U}(1)$ restricted model to the $\mathfrak{s u}(2)$ Cartan generator, which is $\mathbb{P}^{1}$ fibered over the $\mathfrak{s u}(2)$-divisor $\left\{a_{4}=0\right\}$. Since the $\mathrm{U}(1)$ restricted model has only one type of charged singlet, which becomes part of the $\mathfrak{s u}(2)$ adjoint multiplet, the specialization to $a_{3} \equiv 0$ does not give rise to any extra matter states. This way the gauge group $G=\mathrm{SU}(2) / \mathbb{Z}_{2}$ could in fact have been anticipated even without any knowledge of the torsional Mordell-Weil group. The reverse process corresponds to the Higgsing of $G=\mathrm{SU}(2) / \mathbb{Z}_{2}$ to $\mathrm{U}(1)$ via a Higgs in the adjoint of $\mathrm{SU}(2)$, more precisely the component with zero Cartan charge.

A further factorisation $a_{4}=a_{4,1} w$ enhances, as described, the gauge group to $G=$ $(\mathrm{SU}(2) \times \mathrm{SU}(2)) / \mathbb{Z}_{2}$ (cf. 4.29) without changing the Mordell-Weil group. Finally, if $w \in$ $H^{0}\left(\mathcal{B}, \overline{\mathcal{K}}^{2}\right)$, specialising in addition to $a_{2}=-\left(w+a_{4,1}\right)$ enhances the Mordell-Weil group to $\mathbb{Z}_{2} \oplus \mathbb{Z}$ and the gauge group to $G=(\mathrm{SU}(2) \times \mathrm{SU}(2)) / \mathbb{Z}_{2} \times \mathrm{U}(1)$ - see (5.3) with $\gamma_{2}=a_{4,1}$ and $\delta_{2}=w$. The reversed chain of Higgsing thus relates all these fibrations as

$$
\frac{\mathrm{SU}(2) \times \mathrm{SU}(2)}{\mathbb{Z}_{2}} \times \mathrm{U}(1) \rightarrow \frac{\mathrm{SU}(2) \times \mathrm{SU}(2)}{\mathbb{Z}_{2}} \rightarrow \frac{\mathrm{SU}(2)}{\mathbb{Z}_{2}} \rightarrow \mathrm{U}(1) \rightarrow \emptyset .
$$

Note that the fibration (4.29) with $G=(\mathrm{SU}(2) \times \mathrm{SU}(2)) / \mathbb{Z}_{2}$ can be shown to coincide with a model that was recently considered in [20]. In this paper, a different chain of Higgsing was considered which takes the form

$$
\frac{\mathrm{SU}(2) \times \mathrm{SU}(2)}{\mathbb{Z}_{2}} \rightarrow \mathrm{SU}(2) \rightarrow \mathrm{U}(1) \rightarrow \mathbb{Z}_{2}
$$

The chain (5.19) is a specialization of the deformations involved in (5.20). In particular, the fibration with Mordell-Weil group $\mathbb{Z}$ and $G=\mathrm{U}(1)$ reached in (5.20) is described as a special $\mathbb{P}_{1,1,2}[4]$-fibration [9] and can in general not be represented as a global Tate model. However, a specialization of this family of fibrations corresponds to the $\mathrm{U}(1)$ restricted Tate model appearing in (5.19). The endpoint of the Higgsing process (5.20) with gauge group $\mathbb{Z}_{2}$ is a genus-one fibration [65] which is not an elliptic fibration. The absence of a $\mathbb{Z}_{2}$ remnant in the last step in our chain (5.19) can be viewed as a consequence of the division by the $\mathbb{Z}_{2}$ center in the $G=\mathrm{SU}(2) / \mathbb{Z}_{2}$ model.

\section{$5.3 \quad$ An $(\mathrm{SU}(4) \times \mathrm{SU}(2) \times \mathrm{SU}(2)) / \mathbb{Z}_{2} \times \mathrm{U}(1)$ fibration}

We now exemplify the implementation of a further non-abelian singularity by constructing a top. According to the classification in [43] the only A-type singularities admissible over this polygon are $A_{3+2 n}$, in agreement with Tate's algorithm. We consider here the $A_{3}=\mathfrak{s u}(4)$ case, with a unique top corresponding to the dual on the righthand side in figure 7 . The hypersurface equation is given by

$$
\hat{P}=e_{2} e_{3} c^{2} d u^{2} v^{2}+e_{1} e_{2} c d^{2} v^{2} w^{2}+\gamma_{1} c d u v w z+\gamma_{2} e_{0} e_{1} d w^{2} z^{2}+\delta_{2} e_{0} e_{3} c u^{2} z^{2}
$$



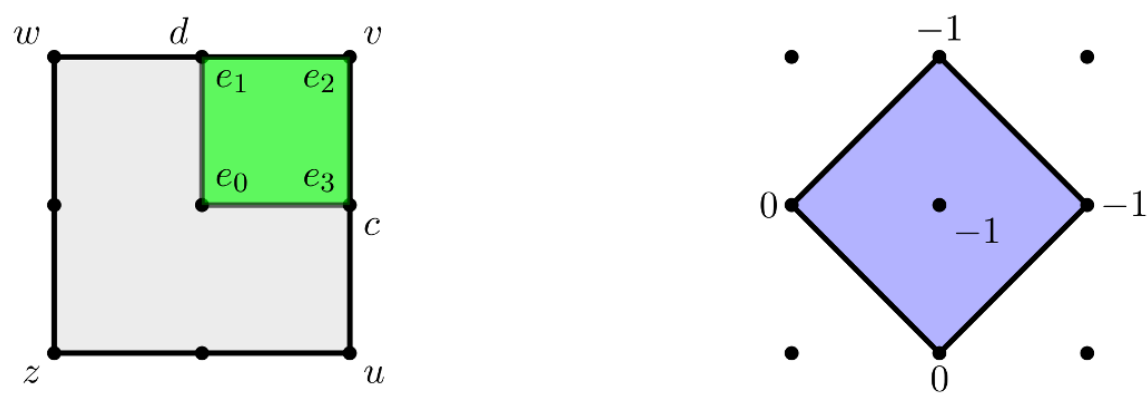

Figure 7. To the left a $\mathfrak{s u}(4)$ top over polygon 15 of [43]. The green layer contains the points at height one. To the right the dual top, bounded from below by the values $z_{\min }$ shown next to the nodes.

with discriminant

$$
\Delta \sim \varpi^{4} \gamma_{2}^{2} \delta_{2}^{2}\left[\gamma_{1}^{4}-8 \varpi \gamma_{1}^{2}\left(\delta_{2}+\gamma_{2}\right)+16 \varpi^{2}\left(\gamma_{2}-\delta_{2}\right)^{2}\right]
$$

for $\pi^{*} \varpi=e_{0} e_{1} e_{2} e_{3}$. We see that imposing the factorization

$$
\gamma_{1} \rightarrow \gamma_{1}, \quad \gamma_{2} \rightarrow \varpi \gamma_{2}, \quad \delta_{2} \rightarrow \varpi \delta_{2}
$$

on the coefficients of (5.1) gives the same behaviour as the top construction. This pattern is just the standard factorisation deduced by the Tate algorithm. For the chosen triangulation of this top we obtain a Stanley-Reisner ideal generated by

$\{u v, u w, u d, v z, z c, z d, w c, c d, v w$

$$
\left.c e_{0}, d e_{0}, v e_{0}, c e_{1}, u e_{1}, z e_{1}, d e_{2}, w e_{2}, z e_{2}, c e_{3}, d e_{3}, v e_{3}, w e_{3}, z e_{3}, e_{1} e_{3}, u e_{0} e_{2}\right\} .
$$

In addition to the $A_{1}$ singularities, with resolution divisors $C$ and $D$, we have a fiber degeneration over $\{\varpi=0\}$ with irreducible components

$$
\mathbb{P}_{i}^{1}=\left\{E_{i}\right\} \cap\left\{\left.\hat{P}\right|_{e_{i}=0}=0\right\} \cap D_{a} \cap D_{b} \quad i=0, \ldots, 3,
$$

where $D_{a}$ and $D_{b}$ are some generic divisors in $\mathcal{B}$. These are intersecting as the affine $\mathfrak{s u}(4)$ Dynkin diagram, as can be read off from the top in figure 7 . For the explicit expressions we refer to appendix A.2. The U(1)-generator from the previous section gets corrected by the exceptional divisors from the extra $\mathfrak{s u}(4)$ locus and takes the form

$$
W_{U}=4(U-Z-\overline{\mathcal{K}})+2 C+E_{1}+2 E_{2}+3 E_{3} .
$$

The normalization is chosen such as to give integer charges of all matter states. In the same way we get additional contributions to the Shioda map $\Sigma_{2}$ of the torsion section, which is a trivial class since $V$ can be written as the linear combination

$$
V=Z+\overline{\mathcal{K}}-\frac{1}{2}\left(C+D+E_{1}+2 E_{2}+E_{3}\right) .
$$

We identify with the new coweight the integer class

$$
\Xi_{2} \equiv V-Z-\overline{\mathcal{K}}=\frac{1}{2}\left(C+D+E_{1}+2 E_{2}+E_{3}\right),
$$


which is 2-torsion in $H^{1,1}\left(\hat{Y}_{4}, \mathbb{Z}\right)$ modulo resolution classes. Repeating the analysis of the previous section we find that the extra coweight class $\Xi_{2}$ is independent of the $\mathrm{U}(1)$-generator.

In what follows we compute the additional charged matter representations localized at codimension-two loci in the base, i.e. the matter curves that lie in the $\mathfrak{s u}(4)$ divisor $\{\varpi=0\}$. The full equations are omitted here and are found appendix A.2. By inspection of the discriminant (5.22) the potential enhancement loci are

$$
\left\{\varpi=\gamma_{1}=0\right\}, \quad\left\{\varpi=\gamma_{2}=0\right\}, \quad\left\{\varpi=\delta_{2}=0\right\},
$$

in addition to the curves considered in the previous section. At $\left\{\varpi=\gamma_{1}=0\right\}$ the fiber components $\mathbb{P}_{0}^{1}$ and $\mathbb{P}_{2}^{1}$ split and the total fiber has the intersection structure of the affine $D_{4}$ Dynkin diagram. The weights of the split curves are

$$
\begin{array}{ll}
\mathbb{P}_{e_{0}=e_{2}=0}^{1} \cdot\left(E_{1}, E_{2}, E_{3}\right)=(1,-1,1), & \mathbb{P}_{e_{0}=e_{2}=0}^{1} \cdot(C, D)=(0,0), \\
\mathbb{P}_{e_{0}=e_{3} u^{2}+e_{1} w^{2}=0}^{1} \cdot\left(E_{1}, E_{2}, E_{3}\right)=(0,1,0), & \mathbb{P}_{e_{0}=e_{3} u^{2}+e_{1} w^{2}=0}^{1} \cdot(C, D)=(0,0), \\
\mathbb{P}_{e_{2}=\gamma_{2} e_{1}+\delta_{2} e_{3} c u^{2}=0}^{1} \cdot\left(E_{1}, E_{2}, E_{3}\right)=(0,-1,0), & \mathbb{P}_{e_{2}=\gamma_{2} e_{1}+\delta_{2} e_{3} c u^{2}=0}^{1} \cdot(C, D)=(0,0)
\end{array}
$$

The $(0,1,0)$ is the highest weight of the $\mathbf{6}$ of $\mathfrak{s u}(4)$. Including the $\mathrm{U}(1)$ charges we therefore find the representation $(\mathbf{6}, \mathbf{1}, \mathbf{1})_{2}+$ c.c..

At $\left\{\varpi=\gamma_{2}=0\right\}$ the curve $\mathbb{P}_{2}^{1}$ splits into three components and the full fiber has the structure of an affine $A_{5}$ Dynkin diagram. We expect to find matter charged under the $\mathfrak{s u}(4)$ and the $\mathfrak{s u}(2)_{C}$ factors along this curve in the base. Indeed the split curves have charges

$$
\begin{array}{ll}
\mathbb{P}_{e_{2}=c=0}^{1} \cdot\left(E_{1}, E_{2}, E_{3}\right)=(0,0,0), & \mathbb{P}_{e_{2}=c=0}^{1} \cdot(C, D)=(-2,0), \\
\mathbb{P}_{e_{2}=u=0}^{1} \cdot\left(E_{1}, E_{2}, E_{3}\right)=(0,-1,1), & \mathbb{P}_{e_{2}=u=0}^{1} \cdot(C, D)=(1,0), \\
\mathbb{P}_{e_{2}=\gamma_{1} v+\delta_{2} e_{0} e_{3} u=0}^{1} \cdot\left(E_{1}, E_{2}, E_{3}\right)=(1,-1,0), & \mathbb{P}_{e_{2}=\gamma_{1} v+\delta_{2} e_{0} e_{3} u=0}^{1} \cdot(C, D)=(1,0),
\end{array}
$$

where the $(0,-1,1)$ and the $(1,-1,0)$ are weights in the fundamentals 4 and $\overline{4}$ respectively. Including the $\mathrm{U}(1)$ charges we have the $(\mathbf{4}, \mathbf{2}, \mathbf{1})_{1}+$ c.c. along this matter curve.

Along $\varpi=\delta_{2}=0$ the configuration is completely analogous to that along $\varpi=\gamma_{2}=0$ and gives rise to massless matter in representation $(\mathbf{4}, \mathbf{1}, \mathbf{2})_{1}+$ c.c.. The massless matter spectrum is summarized in the following table:

\begin{tabular}{|cc|}
\hline \multicolumn{2}{|c|}{ Top over polygon 15: } \\
$\mathfrak{s u}(4) \times \mathfrak{s u}(2)_{C} \times \mathfrak{s u}(2)_{D} \times \mathrm{U}(1)$ \\
\hline Locus & Charged matter \\
$\gamma_{1} \cap\left\{\gamma_{2}=\delta_{2}\right\}$ & $(\mathbf{1}, \mathbf{1}, \mathbf{1})_{4},(\mathbf{1}, \mathbf{1}, \mathbf{1})_{-4}$ \\
$\gamma_{2} \cap \delta_{2}$ & $(\mathbf{1}, \mathbf{2}, \mathbf{2})_{2},(\mathbf{1}, \mathbf{2}, \mathbf{2})_{-2}$ \\
$\varpi \cap \gamma_{1}$ & $(\mathbf{6}, \mathbf{1}, \mathbf{1})_{2},(\mathbf{6}, \mathbf{1}, \mathbf{1})_{-2}$ \\
$\varpi \cap \gamma_{2}$ & $(\mathbf{4}, \mathbf{2}, \mathbf{1})_{1},(\overline{\mathbf{4}}, \mathbf{2}, \mathbf{1})_{-1}$ \\
$\varpi \cap \delta_{2}$ & $(\mathbf{4}, \mathbf{1}, \mathbf{2})_{1},(\overline{\mathbf{4}}, \mathbf{1}, \mathbf{2})_{-1}$ \\
\hline
\end{tabular}


It is confirmed that the coweight element $\Xi_{2}$ is integer-valued on all split curves responsible for the matter representations. We finally conclude that the gauge group is

$$
\frac{\mathrm{SU}(4) \times \mathrm{SU}(2)_{C} \times \mathrm{SU}(2)_{D}}{\mathbb{Z}_{2}} \times \mathrm{U}(1) .
$$

\section{$6 \quad$ Mordell-Weil group $\mathbb{Z}_{3}$}

As a further illustration we now analyze elliptic fibrations with $\mathbb{Z}_{3}$ torsional Mordell-Weil group. The general form of such fibrations was derived in [32]. As we will show this fibration allows for a toric representation, which in fact coincides with the last of the 3 reflexive pairs of polygons admitting a torsional Mordell-Weil group [15]. The fan is given by the 16th reflexive polygon in the enumeration by [43]. We first present the toric representation of this fibration, its singularity structure and impose further non-abelian degenerations of the fiber to analyse the resulting matter spectrum and global structure of the gauge group.

\subsection{An $\mathrm{SU}(3) / \mathbb{Z}_{3}$-fibration}

The generic form of an elliptic fibration with a $\mathbb{Z}_{3}$-section is given by the vanishing locus of the hypersurface equation [32]

$$
P=y^{2}+a_{1} x y z+a_{3} y z^{3}-x^{3}
$$

in weighted projective space $\mathbb{P}_{[2,3,1]}$. Such fibrations therefore fit again into the class of global Tate models, but with $a_{6} \equiv 0$ and in addition $a_{2} \equiv 0$ and $a_{4} \equiv 0$. The equivalent Weierstrass model is defined by

$$
f=\frac{1}{2} a_{1} a_{3}-\frac{1}{48} a_{1}^{4}, \quad g=\frac{1}{4} a_{3}^{2}+\frac{1}{864} a_{1}^{6}-\frac{1}{24} a_{1}^{3} a_{3}
$$

with discriminant

$$
\Delta=\frac{1}{16} a_{3}^{3}\left(27 a_{3}-a_{1}^{3}\right)
$$

The vanishing order of $\Delta$ at $\left\{a_{3}=0\right\}$, where neither $f$ nor $g$ vanish, signals an $A_{2^{-}}$ singularity over this locus. The singularity at $x=y=a_{3}=0$ is resolved by two blow-ups

$$
(x, y) \rightarrow(s x, s y), \quad(s, y) \rightarrow(q s, q y)
$$

with proper transform

$$
\hat{P}=s q^{2} y^{2}+a_{1} q s x y z+a_{3} y z^{3}-q s^{2} x^{3}
$$

as the resulting equation. The Stanley-Reisner ideal after these two blow-ups is $\{q x, q y, q z, x y, s z\}$ (see figure 8). The hypersurface equation (6.1) has an equivalent toric description as a generic hypersurface which makes the vanishing of the coefficients $a_{2}, a_{4}$ and $a_{6}$ manifest. To see this we perform yet another blow-up by

$$
q \rightarrow p q, \quad y \rightarrow p y
$$



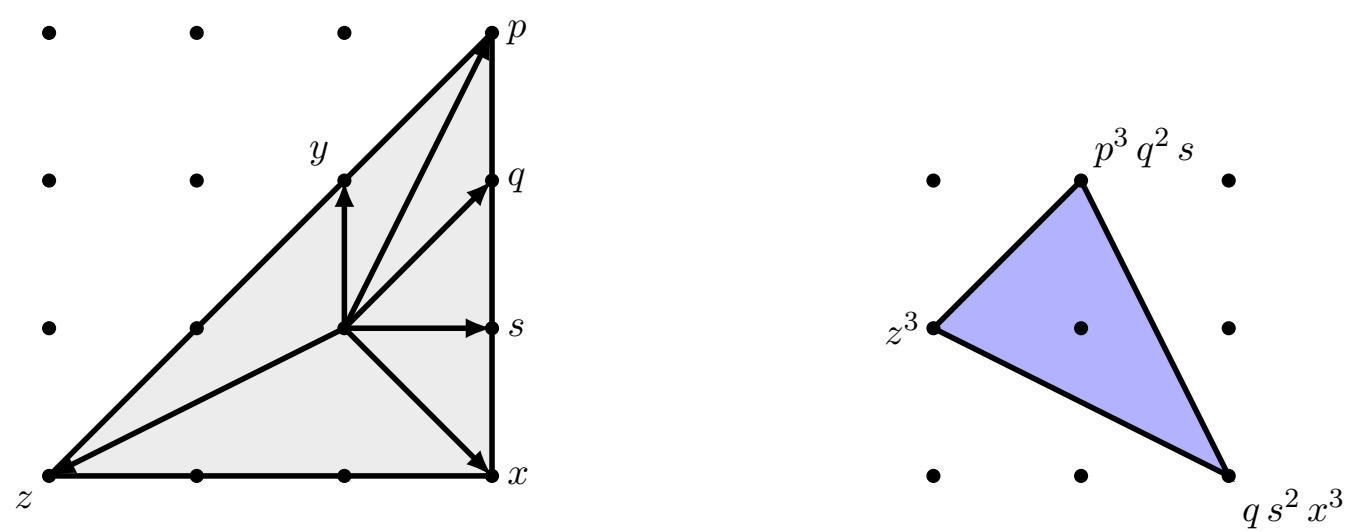

Figure 8. Polygon 16 of [43] together with its dual polygon. The coordinate $y$ is scaled to one and does not contribute to the monomials.

under which the proper transform of equation (6.5) is

$$
\hat{P}=s p^{3} q^{2} y^{2}+a_{1} p q s x y z+a_{3} y z^{3}-q s^{2} x^{3} .
$$

The Stanley-Reisner ideal now extends to $\{s z, q z, p z, x y, s y, q y, p s, p x, q x\}$ and implies that the locus $\{y=0\}$ does not intersect the hypersurface any more. Hence we can use one scaling relation to set $y=1$. After this step we arrive at the hypersurface equation

$$
\hat{P}=p^{3} q^{2} s+a_{1} p q s x z+a_{3} z^{3}-q s^{2} x^{3}
$$

defined in the ambient space with scaling relations

\begin{tabular}{|c|c|c|c|c|c|}
\hline$x$ & $z$ & $s$ & $q$ & $p$ & $\sum$ \\
\hline 1 & 1 & 0 & 0 & 1 & 3 \\
\hline 1 & 2 & 0 & 3 & 0 & 6 \\
\hline 0 & 1 & 1 & 1 & 0 & 3 \\
\hline
\end{tabular}

and SR ideal $\{s z, q z, p x, p s, q x\}$. A blow-down of this fibration was also considered in [41], where it was shown that the structure group of the elliptic fibration is the subgroup $\Gamma_{0}(3)$ of $\operatorname{SL}(2, \mathbb{Z})$. As we will see, the structure of admissible gauge groups is in agreement with the appearance of such restricted monodromy.

Over the locus $\left\{a_{3}=0\right\}$ the hypersurface equation (6.8) factors as

$$
\left.\hat{P}\right|_{a_{3}=0}=q s\left(p^{3} q-s x^{3}-a_{1} p x z\right)
$$

with three irreducible factors. The intersection pattern of the irreducible parts of the fiber, denoted by $\mathbb{P}_{s}^{1}, \mathbb{P}_{q}^{1}$ and $\mathbb{P}_{e q}^{1}$, is shown in figure 9. The two resolution divisors $Q:\{q=0\}$ and $S:\{s=0\}$ are $\mathbb{P}^{1}$-fibrations over $\left\{a_{3}=0\right\}$ and are associated with the two Cartan generators of $\mathfrak{s u}(3)$.

The vanishing order of the discriminant increases by 1 on the curve $\left\{a_{3}=0\right\} \cap\left\{a_{1}=0\right\}$, naively suggesting an enhancement of the singularity type from $A_{2}$ to $A_{3}$ and thus localised matter in the fundamental $\mathbf{3}$ of $\mathfrak{s u}(3)$. In actuality, however, no higher degeneration of the 


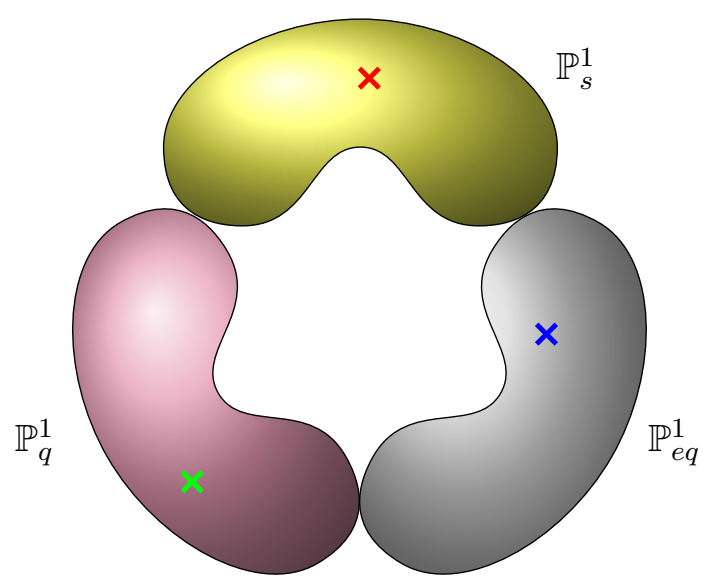

Figure 9. The factorised fiber over the base locus $a_{3}=0$; the blue cross indicates the zero point $z=0$ and the green and red crosses indicates the points $p=0$ and $x=0$, respectively.

fiber structure occurs over this curve because none of the three components in (6.10) factorises further. This can be seen directly by considering the Weierstrass coefficients $f$ and $g$ (6.2): along $\left\{a_{3}=0\right\} \cap\left\{a_{1}=0\right\}$, each coefficient vanishes to order 2 , which implies that the Kodaira type of the degenerate fibers is type $I V$. This is very similar to the familiar $A_{2}$, except that the three components of the fibers meet in a single point rather than meeting pairwise at three different points. There is no enhancement or matter (consistent with $[58,59]$ ). The absence of the fundamental representation, which would be expected to be present in generic fibrations with $\mathfrak{s u}(3)$ gauge algebra, will be understood momentarily from the global structure of the gauge group.

The toric Mordell-Weil group is generated by the differences $P-Z$ or $X-Z$ with $P, X, Z$ corresponding to the vertices of polygon 16 [15] with coordinates as in figure 8 . Using the SR-ideal, we conclude that each of these sections intersects only one of the $\mathbb{P}^{1}$ 's, and each $\mathbb{P}^{1}$ intersects only one of the sections.

The divisor class $Y:\{y=0\}$ does not intersect the hypersurface and may be expressed as

$$
Y=3 Z-S-2 Q-3 P+3 \overline{\mathcal{K}} .
$$

Hence we can define the integer class

$$
\Xi_{3} \equiv P-Z-\overline{\mathcal{K}}=-\frac{1}{3}(S+2 Q)
$$

associated with a new coweight. Any weight of a charged matter representation has to have integer pairing with $\Xi_{3}$, making the weight lattice an order three coarser lattice. In particular, this forbids the fundamental representation of $\mathrm{SU}(3)$, in agreement with our findings above. Note also that the fundamental representation would be transforming under the center $\mathbb{Z}_{3}$ of $\mathrm{SU}(3)$. Thus the gauge group is $\mathrm{SU}(3) / \mathbb{Z}_{3}$.

Note that the specialization $a_{3}=\left(\tilde{a}_{3}\right)^{n}$, if admissible, modifies the gauge group to

$$
G=\mathrm{SU}(3 n) / \mathbb{Z}_{3},
$$



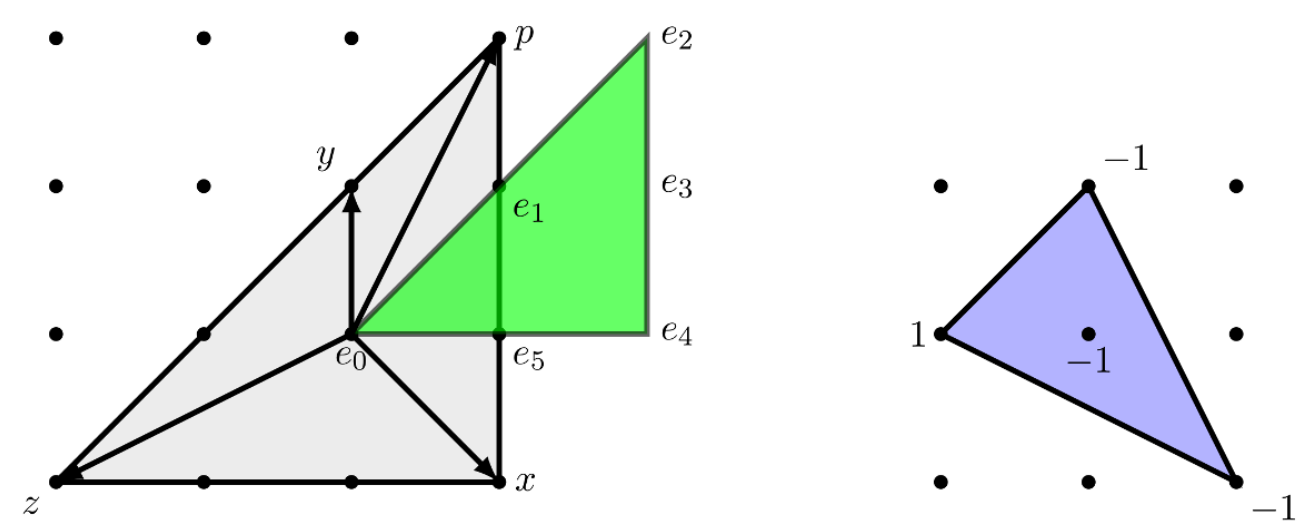

Figure 10. The $\mathfrak{s u}(6)$ top over polygon 16 is shown to the left. The green layer contains the points at height one. The right side defines the dual top, bounded from below by the values $z_{\min }$ shown next to the nodes.

corresponding to a fiber structure of split Kodaira type $I_{3 n}^{s}$. For $n=2$, the fiber over the curve $\left\{\tilde{a}_{3}=0\right\} \cap\left\{a_{1}=0\right\}$ degenerates further to Kodaira type $I V^{*}$, as reflected in the vanishing orders $(3,4,8)$ of $(f, g, \Delta)$ in the Weierstrass model. This signals an enhancement of the singularity type from $A_{5} \simeq \mathfrak{s u}(6)$ to $E_{6}$. From the branching rules of the adjoint representation of $E_{6}$ under the decomposition to $\mathfrak{s u}(6)$ one infers massless matter in the triple-antisymmetric representation $\mathbf{2 0}$ of $\mathfrak{s u}(6)$, in agreement with the gauge group $\mathrm{SU}(6) / \mathbb{Z}_{3}$. However, for $n \geq 3$ the Kodaira type fiber over $\left\{\tilde{a}_{3}=0\right\} \cap\left\{a_{1}=0\right\}$ is beyond $E_{8}$ according to Kodaira's list. This means that no crepant resolution of the fibration exists whenever the locus $\left\{\tilde{a}_{3}=0\right\} \cap\left\{a_{1}=0\right\}$ is non-trivial, and F-theory on such spaces is ill-defined. This complication does not arise for eight-dimensional F-theory compactifications on $\mathrm{K} 3$, where the codimension-one loci are points on the base $\mathcal{B}=\mathbb{P}^{1}$ and thus no problematic enhancement of this type arises. Indeed, the case $n=6$ corresponds to the $\mathrm{SU}(18) / \mathbb{Z}_{3}$ model presented in eq. (5.4) of [62] for F-theory on a K3 surface.

Finally, let us note that the F-theory model does not possess a well-defined weak coupling Type IIB limit, at least not of the usual type à la Sen: since $a_{2} \equiv 0$ (in addition to $a_{4} \equiv 0$ and $a_{6} \equiv 0$ ), the quantity $h$ defining the Type IIB Calabi-Yau $X_{\text {IIB }}$ as the hypersurface $\xi^{2}=h$ factorises, $h=-\frac{1}{12} a_{1}^{2}$. Thus the locus $\xi=0=a_{1}$ is singular.

\subsection{An $(\mathrm{SU}(6) \times \mathrm{SU}(3)) / \mathbb{Z}_{3}$-fibration}

To further illustrate this relation between the $\mathbb{Z}_{3}$ Mordell-Weil group and the global structure of the gauge group we implement an additional non-abelian fiber degeneration in codimension-one. This results in an F-theory compactification with a richer matter spectrum. As we will see, only matter representations occur which are compatible with the extra coweight induced by the torsion generator of the Mordell-Weil group. To implement an extra non-Abelian singularity in the hypersurface (6.8) we construct a top. According to the classification in [43] the only possible tops encoding A-type degenerations are the affine $A_{2}, A_{5}, A_{8}$ etc. Here we construct the single top corresponding to the affine $A_{5}$, realizing an $\mathfrak{s u}(6)$ theory along a divisor in the base. The hypersurface equation in the 
ambient space defined by the top is now given by

$$
\hat{P}=e_{1} e_{2}^{2} e_{3} p^{3} q^{2} s+a_{1} p q s x z+a_{3} e_{0}^{2} e_{1} e_{5} z^{3}-e_{3} e_{4}^{2} e_{5} q s^{2} x^{3},
$$

where the coefficients of the monomials are chosen to match (6.8). The discriminant takes the form

$$
\Delta \sim w^{6} a_{3}^{3}\left(a_{1}^{3}-27 w^{2} a_{3}\right),
$$

where $\pi^{*} w=e_{0} e_{1} e_{2} e_{3} e_{4} e_{5}$ defines the $\mathfrak{s u}(6)$-divisor as $W:\{w=0\}$ in the base $\mathcal{B}$. For the chosen triangulation of the top we obtain the Stanley-Reisner ideal

$$
\begin{aligned}
& \left\{p s, p x, q x, q z, s z, p e_{3}, p e_{4}, p e_{5}, q e_{0}, q e_{1}, q e_{3}, q e_{4}, q e_{5}, z e_{1}, z e_{2}, z e_{3}, z e_{4}, z e_{5},\right. \\
& \left.s e_{0}, s e_{1}, s e_{3}, s e_{4}, s e_{5}, x e_{1}, x e_{0} e_{3}, x e_{3} e_{5}, e_{0} e_{2}, e_{0} e_{4}, e_{1} e_{4}, e_{1} e_{5}, e_{2} e_{4}, e_{2} e_{5}\right\} .
\end{aligned}
$$

In addition to the $A_{2}$ singularity with resolution divisors $S$ and $Q$ one finds a fiber degeneration over $W:\{w=0\}$ with irreducible components

$$
\mathbb{P}_{i}^{1}=\left\{E_{i}\right\} \cap\left\{\left.P_{W}\right|_{e_{i}=0}=0\right\} \cap D_{a} \cap D_{b} \quad i=0, \ldots, 5,
$$

where $D_{a}$ and $D_{b}$ are some generic divisors in $\mathcal{B}$. These are intersecting as the affine $A_{5}$ Dynkin diagram as can also be read off from the top in figure 10. For the explicit expressions we refer to appendix A.3.

We next compute the charged matter representations at enhancement loci in codimension-two. By inspection of the discriminant (6.15) we see that there are three potentially interesting loci,

$$
\left\{w=a_{1}=0\right\}, \quad\left\{w=a_{3}=0\right\} \quad \text { and } \quad\left\{a_{1}=a_{3}=0\right\} .
$$

The locus $\left\{a_{1}=a_{3}=0\right\}$, despite the increased vanishing order of $\Delta$, does not give rise any massless matter, as discussed already in the previous section. Thus, no massless states in representation $(\mathbf{1}, \mathbf{3})$ of $\mathfrak{s u}(6) \oplus \mathfrak{s u}(3)$ exist. The enhancement over the remaining two loci is determined by calculating the factorization of the fiber components over these loci. The explicit equations are presented in appendix A.3.

At $\left\{w=a_{1}=0\right\}$ the fiber components $\mathbb{P}_{0}^{1}$ and $\mathbb{P}_{3}^{1}$ factorize, resulting in six distinct fiber components. They intersect as the non-affine $E_{6}$ Dynkin diagram. The weights at this locus are obtained by computing the intersection numbers of the split fiber components with the resolution divisors $E_{i}$ and $S, Q$ of the $\mathfrak{s u}(6)$ and $\mathfrak{s u}(3)$ singularities, respectively. As an example we consider the split curves arising from $\mathbb{P}_{0}^{1}$ and compute the weights

$$
\begin{array}{ll}
\mathbb{P}_{e_{0}=e_{3}=0}^{1} \cdot\left(E_{1}, E_{2}, E_{3}, E_{4}, E_{5}\right)=(1,0,-1,0,1), & \mathbb{P}_{e_{0}=e_{3}=0}^{1} \cdot(S, Q)=(0,0), \\
\mathbb{P}_{e_{0}=e_{1} p^{3}+e_{5} x^{3}=0}^{1} \cdot\left(E_{1}, E_{2}, E_{3}, E_{4}, E_{5}\right)=(0,0,1,0,0), & \mathbb{P}_{e_{0}=e_{1} p^{3}+e_{5} x^{3}=0}^{1} \cdot(S, Q)=(0,0),
\end{array}
$$

which are in the $(\mathbf{2 0}, \mathbf{1})$ of $\mathfrak{s u}(6) \oplus \mathfrak{s u}(3)$.

Over $\left\{w=a_{3}=0\right\}$ the component $\mathbb{P}_{3}^{1}$ factorizes. This results in 9 distinct curves, intersecting as the affine $\mathfrak{s u}(9)$ Dynkin diagram. We compute the charges

$$
\begin{array}{ll}
\mathbb{P}_{e_{2}=x=0}^{1} \cdot\left(E_{1}, E_{2}, E_{3}, E_{4}, E_{5}\right)=(0,-1,1,0,0), & \mathbb{P}_{e_{2}=x=0}^{1} \cdot(S, Q)=(1,0), \\
\mathbb{P}_{e_{2}=a_{1} p+e_{3} s x^{2}=0}^{1} \cdot\left(E_{1}, E_{2}, E_{3}, E_{4}, E_{5}\right)=(1,-1,0,0,0), & \mathbb{P}_{e_{2}=a_{1} p+e_{3} s x^{2}=0}^{1} \cdot(S, Q)=(0,1),
\end{array}
$$


recognizing the $(0,-1,1,0,0)$ and $(1,-1,0,0,0)$ as a weight of the $\mathbf{6}$ and $\overline{\mathbf{6}}$ of $\mathfrak{s u}(6)$, respectively. Taking into account also the $\mathbf{3}$ and $\overline{\mathbf{3}}$ weights of $\mathfrak{s u}(3)$ on the right one deduces along $\left\{w=a_{3}=0\right\}$ matter in the bifundamental $(\mathbf{6}, \mathbf{3})$ (plus its conjugate).

The matter spectrum is summarized in the following table:

\begin{tabular}{|cc|}
\hline \multicolumn{2}{|c|}{ Top over polygon 16: } \\
$\mathfrak{s u}(6) \times \mathfrak{s u}(3)$
\end{tabular}

Finally we remark that the fibration is non-flat at the codimension-three points $w=a_{1}=$ $a_{3}=0$, where one of the defining equations of the fiber components vanishes identically. This is precisely the intersection locus of the matter curves supporting the $(\mathbf{6}, \mathbf{3})$ and $(\mathbf{2 0}, \mathbf{1})$ representations. The severe degeneration of the fibration at this locus reflects the fact no triple Yukawa coupling can be constructed out of the $\mathbf{2 0}$ (antisymmetric in three indices) together with the $\mathbf{6}$ and the $\overline{\mathbf{6}}$. Thus, in order to make sense out of F-theory compactified on the associated Calabi-Yau 4-fold the matter curves in question must not meet, which is a strong constraint on the base space $\mathcal{B}$. This constraint does not arise for F-theory on lower-dimensional Calabi-Yau $n$-folds.

We are now in a position to discuss the global structure of the gauge group. The Shioda-type map for the generator of the $\mathbb{Z}_{3}$-torsional Mordell-Weil group reads

$$
\Sigma_{3}=P-Z-\overline{\mathcal{K}}+\frac{1}{3}\left(S+2 Q+2 E_{1}+4 E_{2}+3 E_{3}+2 E_{4}+E_{5}\right)
$$

with $\overline{\mathcal{K}}=\pi^{*} \overline{\mathcal{K}}_{\mathcal{B}}$. Here $P=\{p=0\}$, whose intersection with the fiber is the $\mathbb{Z}_{3}$ torsion point. From the $\mathfrak{s u}(6)$ top we infer that the toric divisor class $\{y=0\}$ in the ambient space is expressed as

$$
Y=3 Z-S-2 Q-3 P+3 \overline{\mathcal{K}}-2 E_{1}-4 E_{2}-3 E_{3}-2 E_{4}-E_{5} .
$$

We thus see that

$$
-3 \Sigma_{3}=Y
$$

and $Y$ does not intersect the hypersurface. Hence $\Sigma_{3}$ is trivial in $H^{1,1}\left(Y_{4}, \mathbb{R}\right)$ and

$$
\Xi_{3} \equiv P-Z-\overline{\mathcal{K}}=-\frac{1}{3}\left(S+2 Q+2 E_{1}+4 E_{2}+3 E_{3}+2 E_{4}+E_{5}\right) .
$$

Again, $P-Z-\overline{\mathcal{K}}$ is a 3 -torsion element of $H^{1,1}\left(\hat{Y}_{4}, \mathbb{Z}\right) /\left\langle F_{i}\right\rangle_{\mathbb{Z}}$ for $\left\langle F_{i}\right\rangle_{\mathbb{Z}}$ the lattice spanned by all the exceptional divisors. Furthermore, it is easy to check that $\Xi_{3}$ has integer intersection with all weights computed computed above. Due to the refinement of the coweight lattice the gauge group for this model is thus

$$
G=\frac{\mathrm{SU}(6) \times \mathrm{SU}(3)}{\mathbb{Z}_{3}}
$$

with $\pi_{1}(G)=\mathbb{Z}_{3}$. The correspondingly coarser weight lattice implies that the center $\Lambda / Q$ of the gauge group is trivial. 


\section{Conclusions}

In this work we have analyzed F-theory compactifications on elliptic fibrations with torsional Mordell-Weil group. While non-torsional rational sections give rise to massless U(1) gauge symmetries, the torsional subgroup affects the global structure of the gauge group. In general, the gauge group is of the form $G \times G^{\prime}$, where $G$ is affected by the Mordell-Weil torsion and $G^{\prime}$ may or may not be trivial. As we have argued, the presence of $\mathbb{Z}_{k}$-torsional sections guarantees the existence of a $k$-fractional linear combination of resolution divisors associated with the Cartan generators of $G$ which has integer intersection number with every fiber component. This fractional linear combination can be identified with an element of the coweight lattice of $G$, which is rendered finer by a factor $k$ compared to the universal cover $G_{0}$ of $G$. This enhances the first fundamental group of $G$ by $\mathbb{Z}_{k}$ (compared to $G_{0}$ ), yielding non-simply connected gauge groups. Consistently, the spectrum of allowed matter representations is constrained to the extent that only those elements in the weight lattice are allowed which have an integer pairing with the coweights associated with the Mordell-Weil torsion. An equivalent way of putting this is that the torsional subgroup $\mathbb{Z}_{k_{1}} \oplus \ldots \oplus \mathbb{Z}_{k_{n}}$ of the Mordell-Weil group can be identified with a subgroup of the center of the universal cover group $G_{0}$, and the gauge group of the F-theory compactification is $G_{0} /\left(\mathbb{Z}_{k_{1}} \oplus \ldots \oplus \mathbb{Z}_{k_{n}}\right) \times G^{\prime}$

It might be worthwhile pointing out that the torsional Mordell-Weil group has no particular effect on the structure of the Yukawa couplings between the matter states as such, which is encoded in the fiber type in codimension-three. Contrary to naive expectations, it is thus not relevant to produce e.g. discrete selection rules in the effective action of an F-theory compactification.

We have exemplified this picture for elliptic fibrations with torsional Mordell-Weil group $\mathbb{Z}_{2}$ and $\mathbb{Z}_{3}$, whose defining equation had already been presented in [32]. These fibrations can be analysed torically as hypersurfaces in toric ambient spaces, and, as we have seen, coincide with two out of the 16 possible hypersurface torus fibrations, whose Mordell-Weil group has been computed also in [15]. The third possible hypersurface elliptic fibration with Mordell-Weil torsion, with Modell-Weil group $\mathbb{Z} \oplus \mathbb{Z}_{2}$ [15], turns out to be a further specialization of the $\mathbb{Z}_{2}$-model. All these fibrations are related to a special class of elliptic fibrations with Mordell-Weil group $\mathbb{Z}[4]$ by a chain of (un)Higgsings.

A possible next step would be to study also fibrations with Mordell-Weil group $\mathbb{Z}_{4}$ and higher. The defining Tate model for examples of such fibrations has been given in [32]. It would be interesting to express these fibrations as complete intersections (as opposed to hypersurfaces) or even determinantal varieties and to study their properties at the same level of detail as achieved for the hypersurface models in this article.

An exciting aspect of gauge theories with non-simply connected gauge groups is the physics of non-local operators such as the spectrum of dyonic Wilson line operators. As studied e.g. in [29], the spectrum of such dyonic operators depends on the weight lattice of the gauge group $G$ and of its Langlands dual $G^{*}$. As we have seen, the weight lattice $\Lambda$ of an F-theory compactification on an elliptic fibration is intimately related to the geometry of torsional sections. It would be interesting to investigate further the relation between this 
geometric picture, the spectrum of dyonic Wilson line operators and the global structure of the gauge group in F-theory.

\section{Acknowledgments}

We are indebted to Eran Palti for initial collaboration and for many important discussions. We also thank Ling Lin for many discussions. This work was partially funded by DFG under Transregio 33 'The Dark Universe', and by the US National Science Foundation under grant PHY-1307513.

\section{A More on fiber structures}

\section{A.1 $\mathfrak{s u ( 4 )}$ top over polygon 13}

Here provide the explicit equations for the fiber components of the $(\mathrm{SU}(4) \times \mathrm{SU}(2)) / \mathbb{Z}_{2^{-}}$ model discussed in section 4.3.

\section{A.1.1 Codimension one}

The equations for the fiber components over $\{w=0\} \subset \mathcal{B}$ are

$$
\begin{array}{llr}
e_{0}=0: & e_{1}+a_{1} t z-e_{3} t^{4}=0 & \left(y=s=e_{2}=1\right), \\
e_{1}=0: & a_{1} s t y z-e_{0} e_{2} e_{3} s t^{2} z^{2} a_{2,1}-e_{0}^{2} e_{3} z^{4} a_{4,2}-e_{2}^{2} e_{3} s^{2} t^{4}=0, & \\
e_{2}=0: & e_{1}-e_{3} a_{4,2}+a_{1} t=0 & \left(y=s=z=e_{0}=1\right), \\
e_{3}=0: & e_{1}+a_{1} t=0 & (y=s=z=1) .
\end{array}
$$

Here we impose the SR-ideal (4.39). The four curves $\mathbb{P}_{i}^{1}$ of these divisors intersect like the nodes of the affine Dynkin diagram of $A_{3}$.

\section{A.1.2 Codimension two}

Over $\left\{w=a_{4,2}=0\right\}$ we obtain:

$$
\begin{array}{rlr}
e_{0}=0: & a_{1} t z-e_{3} t^{4}+e_{1}=0 & \left(y=s=e_{2}=1\right), \\
e_{1}=0: & s t \underbrace{\left(e_{0} e_{2} e_{3} t z^{2} a_{2,1}-a_{1} y z+e_{2}^{2} e_{3} s t^{3}\right)}_{R 1}=0, & \\
e_{2}=0: & a_{1} t+e_{1}=0 & \left(y=s=z=e_{0}=1\right), \\
e_{3}=0: & a_{1} t+e_{1}=0 & (y=s=z=1),
\end{array}
$$

and over $\left\{w=a_{1}=0\right\}$ :

$$
\begin{array}{llr}
e_{0}=0: & e_{1}-e_{3} t^{4}=0 \\
e_{1}=0: & e_{3} \underbrace{\left(e_{0} e_{2} s t^{2} z^{2} a_{2,1}+e_{0}^{2} z^{4} a_{4,2}+e_{2}^{2} s^{2} t^{4}\right)}_{R 2}=0, & \left(y=s=e_{2}=1\right), \\
e_{2}=0: & e_{1}-e_{3} a_{4,2}=0 & \\
e_{3}=0: & e_{1}=0 & \left(y=s=z=e_{0}=1\right), \\
& (y=s=z=1) .
\end{array}
$$


Before calculating the weights we analyse the parts $R 1$ and $R 2$ in detail. For $R 1$ one can check that the divisors $\left\{e_{2}=0\right\},\left\{e_{3}=0\right\},\{t=0\}$ and $\{z=0\}$ do not intersect the divisor given by $R 1$ in the toric variety given by the projection along $e_{1}$. Therefore we can rewrite it as

$$
e_{0} a_{2,1}-y a_{1}+s=0
$$

with $e_{0}, y$ and $s$ the homogeneous coordinates of $\mathbb{P}^{2}$. Since (A.4) is a linear equation, we obtain a $\mathbb{P}^{1}$ for the curve given by $e_{1}=0=R 1$. In the case of $R 2$, we find that $\left\{e_{0}=0\right\}$, $\left\{e_{2}=0\right\},\{z=0\}$ and $\{t=0\}$ does not intersect the divisor $R 2$ in the toric variety given by the projection along $e_{1}$. Hence, we rewrite $R 2$ as

$$
s a_{2,1}+a_{4,2}+s^{2}=0,
$$

where $s$ is now the affine coordinate parametrising $\mathbb{C}$ and the remaining homogeneous coordinates $y$ and $e_{3}$ parametrise a $\mathbb{P}^{1}$. Therefore, we obtain two $\mathbb{P}^{1} \mathrm{~s}$ from $R 2$ which are, however, exchanged when going along the matter curve. Around the branch points $\left\{w=a_{1}=a_{4,2}-\frac{1}{4} a_{2,1}^{2}=0\right\}$ the solutions of $s$ to (A.5) are exchanged.

\section{A.2 $\mathfrak{s u}(4)$ top over polygon 15}

This appendix contains more information on the $(\mathrm{SU}(4) \times \mathrm{SU}(2) \times \mathrm{SU}(2)) / \mathbb{Z}_{2} \times \mathrm{U}(1)$ fibration presented in section 5.3.

\section{A.2.1 Codimension one}

The irreducible fiber components over $\{\varpi=0\}$ are:

$$
\begin{aligned}
e_{0}=0: & e_{2} e_{3} u^{2}+e_{1} e_{2} w^{2}+\gamma_{1} u w z=0 & (c=d=v=1), \\
e_{1}=0: & e_{2} d v^{2}+\gamma_{1} d v w+\delta_{2} e_{0}=0 & \left(c=u=z=e_{3}=1\right), \\
e_{2}=0: & \gamma_{1} c u v+\gamma_{2} e_{0} e_{1}+\delta_{2} e_{0} e_{3} c u^{2}=0 & (d=w=z=1), \\
e_{3}=0: & e_{2}+\gamma_{1} u+\gamma_{2} e_{0}=0 & \left(c=d=v=w=z=e_{1}=1\right) .
\end{aligned}
$$

The resolution $\mathbb{P}^{1}$ 's is the intersection of above equations with two generic and independent divisors in the base and they intersect in the pattern of the affine $A_{3}$ Dynkin diagram.

\section{A.2.2 Codimension two}

Over $\left\{\varpi=\gamma_{1}=0\right\}$ the components of the fiber factorizes as

$$
\begin{aligned}
e_{0}=0: & e_{2}\left(e_{3} u^{2}+e_{1} w^{2}\right)=0 & (c=d=v=1), \\
e_{1}=0: & e_{2} d v^{2}+\delta_{2} e_{0}=0 & \left(c=u=z=e_{3}=1\right), \\
e_{2}=0: & e_{0}\left(\gamma_{2} e_{1}+\delta_{2} e_{3} c u^{2}\right)=0 & (d=w=z=1), \\
e_{3}=0: & e_{2}+\gamma_{2} e_{0}=0 & \left(c=d=v=w=z=e_{1}=1\right)
\end{aligned}
$$

and the components intersect as the affine $D_{4}$ Dynkin diagram. 
Over $\left\{\varpi=\gamma_{2}=0\right\}$ the components of the fiber factorizes as

$$
\begin{array}{llr}
e_{0}=0: \quad e_{2} e_{3} u^{2}+e_{1} e_{2} w^{2}+\gamma_{1} u w z=0 & (c=d=v=1), \\
e_{1}=0: & e_{2} d v^{2}+\gamma_{1} d v w+\delta_{2} e_{0}=0 & \left(c=u=z=e_{3}=1\right), \\
e_{2}=0: & c u\left(\gamma_{1} v+\delta_{2} e_{0} e_{3} u\right)=0 & (d=w=z=1), \\
e_{3}=0: & e_{2}+\gamma_{1} u=0 & \left(c=d=v=w=z=e_{1}=1\right)
\end{array}
$$

with the intersection structure given by the affine $A_{5}$ Dynkin diagram.

Over $\left\{\varpi=\delta_{2}=0\right\}$ the components of the fiber factorizes as

$$
\begin{array}{rlrl}
e_{0}=0: e_{2} e_{3} u^{2}+e_{1} e_{2} w^{2}+\gamma_{1} u w z=0 & (c=d=v=1), \\
e_{1}=0: & d v\left(e_{2} v+\gamma_{1} w=0\right) & \left(c=u=z=e_{3}=1\right), \\
e_{2}=0: & \gamma_{1} c u v+\gamma_{2} e_{0} e_{1}=0 & (d=w=z=1), \\
e_{3}=0: e_{2}+\gamma_{1} u+\gamma_{2} e_{0}=0 & \left(c=d=v=w=z=e_{1}=1\right)
\end{array}
$$

intersecting as the affine $A_{5}$ Dynkin diagram.

\section{A.3 $\mathfrak{s u}(6)$ top over polygon 16}

The fiber structure of the $(\mathrm{SU}(6) \times \mathrm{SU}(3)) / \mathbb{Z}_{3}$-fibration of section 6.2 can be summarized as follows:

\section{A.3.1 Codimension one}

The irreducible fiber components over $\{w=0\}$ take the form

$$
\begin{array}{llrl}
e_{0}=0: & e_{1} e_{3} p^{3}+e_{3} e_{5} x^{3}+a_{1} p x z=0 & \left(s=q=e_{2}=e_{4}=1\right), \\
e_{1}=0: e_{3}+a_{1} p=0 & \left(x=s=q=z=e_{4}=e_{5}=1\right), \\
e_{2}=0: & a_{3} e_{1}+a_{1} p q s x+e_{3} q s^{2} x^{3}=0 & \left(z=e_{0}=e_{4}=e_{5}=1\right), \\
e_{3}=0: & a_{3} e_{0}^{2} e_{1} e_{5}+a_{1} x=0 & (s=q=p=z=1) \\
e_{4}=0: & e_{3}+a_{3} e_{5}+a_{1} x=0 & \left(s=q=p=z=e_{0}=e_{1}=e_{2}=1\right) \\
e_{5}=0: & e_{3}+a_{1} x=0 & \left(s=q=p=z=e_{1}=e_{2}=1\right) .
\end{array}
$$

The resolution $\mathbb{P}^{1}$ 's is the intersection of above equations with two generic and independent divisors in the base and they intersect in the pattern of the affine $A_{5}$ Dynkin diagram.

\section{A.3.2 Codimension two}

Over $\left\{w=a_{1}=0\right\}$ the components of the fiber takes the form

$$
\begin{aligned}
e_{0}=0: & e_{3}\left(e_{1} p^{3}+e_{5} x^{3}\right)=0 & \left(s=q=e_{2}=e_{4}=1\right), \\
e_{1}=0: & e_{3}=0 & \left(x=y=s=q=z=e_{4}=e_{5}=1\right), \\
e_{2}=0: & a_{3} e_{1}+e_{3} q s^{2} x^{3}=0 & \left(y=z=e_{0}=e_{4}=e_{5}=1\right), \\
e_{3}=0: & a_{3} e_{0}^{2} e_{1} e_{5}=0 & (y=s=q=p=z=1) \\
e_{4}=0: & e_{3}+a_{3} e_{5}=0 & \left(y=s=q=z=e_{0}=e_{1}=e_{2}=1\right) \\
e_{5}=0: & e_{3}=0 & \left(y=s=q=p=z=e_{1}=e_{2}=1\right)
\end{aligned}
$$

resulting in 6 distinct $\mathbb{P}^{1}$ 's, intersecting as the $E_{6}$ Dynkin diagram (not affine). 
Over $\left\{w=a_{3}=0\right\}$ the components of the fiber takes the form

$$
\begin{aligned}
e_{0}=0: & e_{1} e_{3} p^{3}+e_{3} e_{5} x^{3}+a_{1} p x z=0 & \left(s=q=e_{2}=e_{4}=1\right), \\
e_{1}=0: & e_{3}+a_{1} p=0 & \left(x=y=s=q=z=e_{4}=e_{5}=1\right), \\
e_{2}=0: & q s x\left(a_{1} p+e_{3} s x^{2}\right)=0 & \left(y=z=e_{0}=e_{4}=e_{5}=1\right), \\
e_{3}=0: & a_{1} x=0 & (y=s=q=p=z=1) \\
e_{4}=0: & e_{3}+a_{1} x=0 & \left(y=s=q=z=e_{0}=e_{1}=e_{2}=1\right) \\
e_{5}=0: & e_{3}+a_{1} x=0 & \left(y=s=q=p=z=e_{1}=e_{2}=1\right)
\end{aligned}
$$

resulting in 9 distinct $\mathbb{P}^{1}$ 's, intersecting as the affine $A_{8}$ Dynkin diagram.

Open Access. This article is distributed under the terms of the Creative Commons Attribution License (CC-BY 4.0), which permits any use, distribution and reproduction in any medium, provided the original author(s) and source are credited.

\section{References}

[1] D.R. Morrison and C. Vafa, Compactifications of F-theory on Calabi-Yau threefolds. 1, Nucl. Phys. B 473 (1996) 74 [hep-th/9602114] [INSPIRE].

[2] D.R. Morrison and C. Vafa, Compactifications of F-theory on Calabi-Yau threefolds. 2, Nucl. Phys. B 476 (1996) 437 [hep-th/9603161] [InSPIRE].

[3] A. Klemm, P. Mayr and C. Vafa, BPS states of exceptional noncritical strings, hep-th/9607139 [INSPIRE].

[4] T.W. Grimm and T. Weigand, On abelian gauge symmetries and proton decay in global F-theory GUTs, Phys. Rev. D 82 (2010) 086009 [arXiv: 1006. 0226] [inSPIRE].

[5] A.P. Braun, A. Collinucci and R. Valandro, G-flux in F-theory and algebraic cycles, Nucl. Phys. B 856 (2012) 129 [arXiv:1107.5337] [INSPIRE].

[6] S. Krause, C. Mayrhofer and T. Weigand, $G_{4}$ flux, chiral matter and singularity resolution in F-theory compactifications, Nucl. Phys. B 858 (2012) 1 [arXiv:1109.3454] [INSPIRE].

[7] T.W. Grimm and H. Hayashi, F-theory fluxes, chirality and Chern-Simons theories, JHEP 03 (2012) 027 [arXiv: 1111.1232] [INSPIRE].

[8] S. Krause, C. Mayrhofer and T. Weigand, Gauge fluxes in F-theory and type IIB orientifolds, JHEP 08 (2012) 119 [arXiv:1202.3138] [INSPIRE].

[9] D.R. Morrison and D.S. Park, F-Theory and the Mordell-Weil group of elliptically-fibered Calabi-Yau threefolds, JHEP 10 (2012) 128 [arXiv:1208.2695] [INSPIRE].

[10] M. Cvetič, T.W. Grimm and D. Klevers, Anomaly cancellation and abelian gauge symmetries in F-theory, JHEP 02 (2013) 101 [arXiv: 1210.6034] [INSPIRE].

[11] C. Mayrhofer, E. Palti and T. Weigand, U(1) symmetries in F-theory GUTs with multiple sections, JHEP 03 (2013) 098 [arXiv:1211.6742] [INSPIRE].

[12] V. Braun, T.W. Grimm and J. Keitel, New global F-theory GUTs with U(1) symmetries, JHEP 09 (2013) 154 [arXiv: 1302.1854] [INSPIRE]. 
[13] J. Borchmann, C. Mayrhofer, E. Palti and T. Weigand, Elliptic fibrations for $\mathrm{SU}(5) \times \mathrm{U}(1) \times \mathrm{U}(1)$ F-theory vacua, Phys. Rev. D 88 (2013) 046005 [arXiv:1303.5054] [INSPIRE].

[14] M. Cvetič, D. Klevers and H. Piragua, F-theory compactifications with multiple U(1)-factors: constructing elliptic fibrations with rational sections, JHEP 06 (2013) 067 [arXiv: 1303.6970] [INSPIRE].

[15] V. Braun, T.W. Grimm and J. Keitel, Geometric engineering in toric F-theory and GUTs with U(1) gauge factors, JHEP 12 (2013) 069 [arXiv: 1306. 0577] [INSPIRE].

[16] M. Cvetič, A. Grassi, D. Klevers and H. Piragua, Chiral four-dimensional F-theory compactifications with $\mathrm{SU}(5)$ and multiple U(1)-factors, JHEP 04 (2014) 010 [arXiv: 1306.3987] [INSPIRE].

[17] J. Borchmann, C. Mayrhofer, E. Palti and T. Weigand, SU(5) tops with multiple U(1)s in F-theory, Nucl. Phys. B 882 (2014) 1 [arXiv:1307.2902] [inSPIRE].

[18] M. Cvetič, D. Klevers and H. Piragua, F-theory compactifications with multiple U(1)-factors: addendum, JHEP 12 (2013) 056 [arXiv:1307.6425] [INSPIRE].

[19] M. Cvetič, D. Klevers, H. Piragua and P. Song, Elliptic fibrations with rank three Mordell-Weil group: F-theory with $\mathrm{U}(1) \times \mathrm{U}(1) \times \mathrm{U}(1)$ gauge symmetry, JHEP 03 (2014) 021 [arXiv: 1310.0463] [INSPIRE].

[20] D.R. Morrison and W. Taylor, Sections, multisections and U(1) fields in F-theory, arXiv: 1404.1527 [INSPIRE].

[21] G. Martini and W. Taylor, 6D F-theory models and elliptically fibered Calabi-Yau threefolds over semi-toric base surfaces, arXiv:1404.6300 [INSPIRE].

[22] N.C. Bizet, A. Klemm and D.V. Lopes, Landscaping with fluxes and the E8 Yukawa Point in F-theory, arXiv:1404.7645 [INSPIRE].

[23] J. Marsano, N. Saulina and S. Schäfer-Nameki, Compact F-theory GUTs with U(1)(PQ), JHEP 04 (2010) 095 [arXiv: 0912.0272] [INSPIRE].

[24] H. Hayashi, T. Kawano, Y. Tsuchiya and T. Watari, More on dimension-4 proton decay problem in F-theory - Spectral surface, discriminant locus and monodromy, Nucl. Phys. B 840 (2010) 304 [arXiv: 1004.3870] [INSPIRE].

[25] M.J. Dolan, J. Marsano, N. Saulina and S. Schäfer-Nameki, F-theory GUTs with U(1) symmetries: generalities and survey, Phys. Rev. D 84 (2011) 066008 [arXiv:1102.0290] [INSPIRE].

[26] M.J. Dolan, J. Marsano and S. Schäfer-Nameki, Unification and phenomenology of F-theory GUTs with U(1)PQ, JHEP 12 (2011) 032 [arXiv:1109.4958] [INSPIRE].

[27] J. Marsano, N. Saulina and S. Schäfer-Nameki, On G-flux, M5 instantons and U(1)s in F-theory, arXiv:1107.1718 [INSPIRE].

[28] A. Maharana and E. Palti, Models of particle physics from type IIB string theory and F-theory: a review, Int. J. Mod. Phys. A 28 (2013) 1330005 [arXiv:1212.0555] [InSPIRE].

[29] O. Aharony, N. Seiberg and Y. Tachikawa, Reading between the lines of four-dimensional gauge theories, JHEP 08 (2013) 115 [arXiv:1305.0318] [INSPIRE].

[30] G. McCabe, The structure and interpretation of the standard model, Elsevier, THe Netherlands (2007). 
[31] J.C. Baez and J. Huerta, The algebra of grand unified theories, Bull. Am. Math. Soc. 47 (2010) 483 [arXiv:0904.1556] [INSPIRE].

[32] P.S. Aspinwall and D.R. Morrison, Nonsimply connected gauge groups and rational points on elliptic curves, JHEP 07 (1998) 012 [hep-th/9805206] [INSPIRE].

[33] P.S. Aspinwall, S.H. Katz and D.R. Morrison, Lie groups, Calabi-Yau threefolds and F-theory, Adv. Theor. Math. Phys. 4 (2000) 95 [hep-th/0002012] [InSPIRE].

[34] M. Fukae, Y. Yamada and S.-K. Yang, Mordell-Weil lattice via string junctions, Nucl. Phys. B 572 (2000) 71 [hep-th/9909122] [INSPIRE].

[35] K. Oguiso and T. Shioda, The Mordell-Weil lattice of a rational elliptic surface, Comment. Math. Univ. St. Paul 40 (1991) 83.

[36] I. Shimada, On elliptic K3 surfaces, Michigan Math. J. 47 (2000) 423, [math. AG/0505140].

[37] Z. Guralnik, String junctions and nonsimply connected gauge groups, JHEP 07 (2001) 002 [hep-th/0102031] [INSPIRE].

[38] T. Shioda, Mordell-Weil Lattices and Galois Representation. I, Proc. Japan Acad. A 65 (1989) 268.

[39] R. Wazir, Arithmetic on elliptic threefolds, Compos. Math. 140 (2001) 567 [math/0112259].

[40] D.S. Park, Anomaly equations and intersection theory, JHEP 01 (2012) 093 [arXiv:1111.2351] [INSPIRE].

[41] P. Berglund, A. Klemm, P. Mayr and S. Theisen, On type IIB vacua with varying coupling constant, Nucl. Phys. B 558 (1999) 178 [hep-th/9805189] [INSPIRE].

[42] P. Candelas, E. Perevalov and G. Rajesh, Comments on A, B, C chains of heterotic and type-II vacua, Nucl. Phys. B 502 (1997) 594 [hep-th/9703148] [INSPIRE].

[43] V. Bouchard and H. Skarke, Affine Kac-Moody algebras, CHL strings and the classification of tops, Adv. Theor. Math. Phys. 7 (2003) 205 [hep-th/0303218] [INSPIRE].

[44] J.H. Silverman, The arithmetic of elliptic curves, Springer, Germany (2008).

[45] S. Lang, Fundamentals of diophantine geometry, Springer, Germany (1983).

[46] S. Lang and A. Néron, Rational points of abelian varieties over function fields, Amer. J. Math. 81 (1959) 95.

[47] R. Kloosterman, On the classification of degree 1 elliptic threefolds with constant j-invariant, Illinois J. Math. 55 (2011) 771.

[48] A. Grassi and V. Perduca, Weierstrass models of elliptic toric K3 hypersurfaces and symplectic cuts, Adv. Theor. Math. Phys. 17 (2013) 741 [arXiv:1201.0930] [InSPIRE].

[49] T. Shioda, On elliptic modular surfaces, J. Math. Soc. Japan 24 (1972) 20.

[50] D. A. Cox and S. Zucker, Intersection numbers of sections of elliptic surfaces, Inv. Math. 53 (1979) 1.

[51] T.W. Grimm, M. Kerstan, E. Palti and T. Weigand, Massive abelian gauge symmetries and fluxes in F-theory, JHEP 12 (2011) 004 [arXiv:1107.3842] [INSPIRE].

[52] A.P. Braun, A. Collinucci and R. Valandro, The fate of U(1)'s at strong coupling in F-theory, JHEP 07 (2014) 028 [arXiv: 1402.4054] [inSPIRE]. 
[53] M.R. Douglas, D.S. Park and C. Schnell, The Cremmer-Scherk mechanism in F-theory compactifications on K3 manifolds, JHEP 05 (2014) 135 [arXiv:1403.1595] [INSPIRE].

[54] H. Jockers and J. Louis, D-terms and F-terms from D7-brane fluxes, Nucl. Phys. B 718 (2005) 203 [hep-th/0502059] [INSPIRE].

[55] M. Buican, D. Malyshev, D.R. Morrison, H. Verlinde and M. Wijnholt, D-branes at singularities, compactification and hypercharge, JHEP 01 (2007) 107 [hep-th/0610007] [INSPIRE].

[56] D. Bump, Lie groups, $2^{\text {nd }}$ edition, Springer, Germany (2013).

[57] P. DiFrancesco, P. Mathieu and D. Senechal, Conformal field theory, Springer, Germany (1997).

[58] M. Bershadsky et al., Geometric singularities and enhanced gauge symmetries, Nucl. Phys. B 481 (1996) 215 [hep-th/9605200] [INSPIRE].

[59] A. Grassi and D.R. Morrison, Anomalies and the Euler characteristic of elliptic Calabi-Yau threefolds, Commun. Num. Theor. Phys. 6 (2012) 51 [arXiv:1109.0042] [inSPIRE].

[60] S. Katz, D.R. Morrison, S. Schäfer-Nameki and J. Sully, Tate's algorithm and F-theory, JHEP 08 (2011) 094 [arXiv: 1106. 3854] [INSPIRE].

[61] P. Candelas and A. Font, Duality between the webs of heterotic and type-II vacua, Nucl. Phys. B 511 (1998) 295 [hep-th/9603170] [INSPIRE].

[62] O.J. Ganor, D.R. Morrison and N. Seiberg, Branes, Calabi-Yau spaces and toroidal compactification of the $N=1$ six-dimensional $E_{8}$ theory, Nucl. Phys. B 487 (1997) 93 [hep-th/9610251] [INSPIRE].

[63] A. Sen, Orientifold limit of F-theory vacua, Phys. Rev. D 55 (1997) 7345 [hep-th/9702165] [INSPIRE].

[64] R. Donagi and M. Wijnholt, Higgs bundles and UV completion in F-theory, Commun. Math. Phys. 326 (2014) 287 [arXiv: 0904.1218] [INSPIRE].

[65] V. Braun and D.R. Morrison, F-theory on genus-one fibrations, JHEP 08 (2014) 132 [arXiv: 1401.7844] [INSPIRE]. 